\title{
A new warmstarting strategy for the primal-dual column generation method
}

\author{
Jacek Gondzio • Pablo González-Brevis
}

Received: date / Accepted: date

\begin{abstract}
This paper presents a new warmstarting technique in the context of a primal-dual column generation method applied to solve a particular class of combinatorial optimization problems. The technique relies on calculating an initial point and on solving auxiliary linear optimization problems to determine the step direction needed to fully restore primal and dual feasibilities after new columns arrive. Conditions on the maximum size of the cuts and on a suitable initial point are discussed. Additionally, the strategy ensures that the duality gap of the warmstart is bounded by the old duality gap multiplied with a (small) constant, which depends on the relation between the old and modified problems. Computational experiments demonstrate the gains achieved when compared to a coldstart approach.
\end{abstract}

Keywords interior point methods - warmstarting · column generation · linear programming $\cdot$ cutting stock problem $\cdot$ vehicle routing problem with time windows

Mathematics Subject Classification (2000) 90C51, 90C05, 65K05, $49 \mathrm{M} 20$

J. Gondzio · P. González-Brevis

School of Mathematics, Maxwell Institute, The University of Edinburgh,Mayfield Road, Edinburgh, EH9 3JZ, UK

E-mail: P.Gonzalez-Brevis@sms.ed.ac.uk

J. Gondzio

E-mail: J.Gondzio@ed.ac.uk

http://www.maths.ed.ac.uk/ gondzio/

P. González-Brevis

Facultad de Ingeniería, Universidad del Desarrollo, Av. Sanhueza 1750, Pedro de Valdivia, Concepción, Chile

E-mail: pablogonzalez@ingenieros.udd.cl 


\section{Introduction}

Starting with the seminal work of Karmarkar [23] the interest and developments in interior point methods (IPMs) have continued over the last decades. Among these methods, a family of primal-dual ones have proven to be the most important and widely used [36]. Despite two decades of successful research concerning this class of methods, there still exists an open question when solving consecutive (closely) related problems: how to efficiently warmstart?

The need of solving subsequent and (closely) related linear programming problems arises in many situations. In numerous applications the instance data is subject to variations and therefore, taking advantage of the previously solved problem could lead to savings in solving the modified problem. Some examples can be found in marketing plans with changing demands or customer preferences or in engineering design with modifying product specifications. For these examples, only the data is modified but the dimension of the problem remains the same. Other examples of such situation arise when column generation, cutting plane methods or branching techniques are used in the context of integer programming. Each of these aforementioned situations is based on solving related subproblems iteratively until some conditions hold. The differences between two subsequent problems under these schemes are the addition or elimination of variables/constraints. Under any of these schemes one could either consider that the size of the problem (number of variables/constraints) is modified from one iteration to another or that the coefficients of a whole row or column are set to zero in one problem but not in the other. In what follows, we consider the former approach, where the sizes of two subsequent problems are different.

In this paper a new warmstarting technique applicable in the context of primal-dual column generation method is presented. A thorough theoretical analysis of the new approach is performed and a computational experience is given to provide evidence of its good behaviour in practice.

Warmstarting is understood as the use of previous information gathered in the solution process of a given problem in order to solve a subsequent related problem. The aim of a warmstarting strategy is to solve the modified problem more efficiently than when no prior information is considered, known as the coldstart approach. The difficulty of interior point methods to re-optimize when compared to active set methods is a well-known issue [1]. It results from a completely different way in which IPMs approach optimality. To guarantee fast convergence IPMs traverse the interior of the feasible set (by exploiting the notion of central path and forcing the iterates to stay in its neighbourhood) and approach the boundary of the feasible region only close to termination. Operating in the interior of the feasible set is the great advantage of IPMs responsible for their spectacular efficiency [36]. However it becomes a curse if one tries a naive warmstarting of IPMs. In general IPMs should not warmstart from the optimal solution of the previous problem because such point is too close to the boundary of the feasible set and is very likely to be far away from the new central path (leading to the optimal solution of the new problem) 
[14]. To overcome these difficulties, several strategies have been proposed in the literature.

\subsection{Literature review}

Freund [9] has proposed a shifted barrier method for solving linear programs in standard-form from an infeasible warmstart. He has shown that under suitable assumptions his potential reduction algorithm runs in polynomial time. In the same spirit as Freund, Benson and Shanno [1] have presented an exact primaldual penalty approach. The authors have introduced a method which relaxes the non-negativity constraints of primal and dual problems and penalizes for any violation of them. Their exact method accounts for small perturbations and requires the setting of some penalization parameters which may be a nontrivial task. A similar approach has been presented in [6]. The authors have reformulated the primal-dual pair using a slacked form (without penalization terms). Theoretical conditions under which warmstarted IPMs perform better than coldstart IPMs are presented. The authors have tested their approach when the original data is perturbed (linear programming) and in a cutting plane context (combinatorial optimization). One of the main advantages of the proposed strategy is that it does not require extensive setting of parameters and is readily applicable to a wide variety of problems. Note that the methods presented in $[1,6,9]$ modify the original problem adding auxiliary variables. These methods gradually drive the auxiliary variables to zero obtaining the optimal solution of the original problem. Another class of warmstarting methods calculates a warmstarting point and re-optimizes the modified problem from this point.

Mitchell [27] has studied different strategies to obtain an interior point when adding cutting planes and variables to a linear problem. His methods rely on calculating directions based on the projection onto the null space of the constraint matrix. A positive step length is always available so the methods recover feasibility in one step. The methods have been implemented using the primal projective standard-form variant of Karmarkar's algorithm for linear programming within a cutting plane method applied to matching problems $[27,29]$. Additionally, Mitchell and Borchers [28] have studied a different and practical warmstarting strategy using the primal-dual barrier method applied to solve linear ordering problems.

Goffin and Vial [12] have studied how to deal with the addition of multiple cuts in a cutting plane framework and have proposed a way of restarting the analytic centre cutting plane method [11]. The strategy assumes that the cuts are central using a Dikin's ellipsoid as a reference and the determination of directions to recover feasibility requires to solve a non-linear optimization problem. The authors have provided bounds on the number of steps to recover an analytic centre which depends on the number of cuts added. Similarly, Oskoorouchi et al. [31] can ensure that dual feasibility is recovered under the condition that the constraints added are moderately deep. As stated in [12], if 
there is at least one deep constraint (constraint which does not intersect the Dikin's ellipsoid around the query point), the proposed method recovers only primal feasibility.

Fliege [8] has proposed a new approach to solve convex multicriteria optimization problems. Having two closely related problems, the method applies few centering steps to the old iterate, so the new point is very close to the new central path. The author shows that to compute a finite set of discrete points to describe the solution set, his method runs in polynomial time. Note that this approach has been studied considering changes only in the objective function coefficients which differs from our case.

Yildirim and Wright [37] have analysed different criteria to determine a well-suited warmstarting point. They have compared several different ways of direction searching (i.e., (weighted) least-square approach and Newton step) and provided complexity and convergence results for each of these methods after perturbing the instance data. The authors have determined the size of the perturbation that can be absorbed by each of these methods. Numerical experiments and further developments in this direction have been presented by John and Yildirim in [21].

A different approach has been presented by Gondzio [14] in a primal-dual cutting plane method context. The author has proposed to fix the initial values of the new components and then restore independently primal and dual feasibilities. To calculate the warmstarting point, modified Newton steps have to be performed. The author has distinguished between deep and shallow cuts adjusting his strategy accordingly. Although a successful implementation has been developed, no theoretical guarantee for its performance has been provided.

In [16], Gondzio and Grothey have introduced a primal-dual interior point method that relies on multiple-centrality corrector techniques to find a warmstarting solution. From this point, their method seeks feasibility. The authors have shown under which conditions their short and long step path following methods can absorb data perturbations in one step or in few steps (for larger perturbations). They have extended their analysis to problems with special structures (primal and dual block angular structures). The same authors have studied an unblocking strategy based on sensitivity analysis [17]. The main idea is to overcome the blocking issue that any advanced starting point may suffer from by increasing the size of the step allowed in the Newton direction. First, the proposed strategy identifies which components the blocking originates from. Then, the method aims to remove the blocking components using sensitivity analysis. Conditions that an unblocking direction must satisfy and proofs of the existence of such direction have been provided. The authors have observed that to absorb more infeasibility in one step it is advantageous to use well-centred points and to keep a large value of the duality measure.

A very different warmstarting approach has recently been proposed by Skajaa et al.[33] who employ the self-dual embedding linear programming model which facilitates taking any point as a warmstarting candidate, including points close to the boundary of the feasible region. 
In $[6,14]$ numerical experiments have been carried out to demonstrate how efficient warmstarting methods are when compared to coldstart in a cutting plane framework and encouraging results have been provided.

\subsection{Context, motivation and contributions}

In this paper we will focus on the case when warmstarting is applied in column generation, specifically in the context of the primal-dual column generation method (PDCGM) described in [15]. We address a particularly important application of the column generation method which arises when solving combinatorial optimization problems.

In [15], the warmstarting strategy proposed in [14] and further improved by heuristic procedures has been used, showing encouraging results. We will describe this strategy later in the paper because it provides a benchmark to our new warmstarting technique. Although the old approach introduced in [14] has worked well in practice, it does not guarantee a full feasibility restoration nor study the quality of the warmstarted iterate after new columns/cuts are added. The main motivation of the design of a new warmstarting strategy presented in this paper is to close this theoretical gap.

Under suitable assumptions, the new algorithm restores primal and dual feasibilities after the addition of new columns. The method relies on two linear optimization problems to calculate the direction which recovers primal and dual feasibilities in one step. The direction is determined so that the small components at a particular solution are not largely modified. The strategy allows to have control over the new duality gap by expanding the neighbourhood of the central path. The analysis is performed for the symmetric neighbourhood of the central path. Conditions are derived for a suitable warmstarting point such that for arbitrarily deep cuts in the dual space (columns in the primal) primal and dual feasibilities are restored in one step. An extra care is taken of the "centrality" of the new point and conditions which guarantee that the new iterate belongs to an expanded symmetric neighbourhood of the central path for the new problem are established. In summary, unlike the method proposed in [14] which was based on a heuristic, the method proposed in this paper has solid theoretical foundations. Computational experiments show that the method is comparable to the method in [14] and in many cases superior to coldstart.

The structure of the remaining sections in this paper is the following. In Section 2, we review the fundamentals of primal-dual methods. In Section 3, we briefly describe column generation methods and its primal-dual version. In Section 4, we present our specialized warmstarting idea providing a theoretical analysis which demonstrates how the algorithm deals with infeasibilities and the proximity to the central path. Computational experiments for solving the root node of the cutting stock problem and the vehicle routing problem with time windows after applying Dantzig-Wolfe decomposition comparing the pro- 
posed strategy with a coldstart strategy are presented in Section 5. In Section 6 the main contributions of this study are summarized.

\section{Primal-dual interior point methods}

In this section we introduce the notation used along the paper and the fundamental ideas behind primal-dual interior point methods. For a more detailed description of this IPM variant, we refer the reader to the textbook of Wright about IPMs [36].

Let us consider a linear programming problem represented by the following primal-dual pair

$$
\begin{aligned}
& \mathcal{P}_{0}:=\min \quad c^{T} x, \quad \text { s.t. } A x=b, \quad x \geq 0, \\
& \mathcal{D}_{0}:=\max \quad b^{T} y, \quad \text { s.t. } A^{T} y+s=c, \quad s \geq 0,
\end{aligned}
$$

where $x \in \mathbb{R}^{n}$ is the vector of primal variables, $y \in \mathbb{R}^{m}$ and $s \in \mathbb{R}^{n}$ are the vectors of dual variables. $A \in \mathbb{R}^{m \times n}$ represents the coefficient matrix, where $\operatorname{rank}(A)=m \leq n$, and $c \in \mathbb{R}^{n}$ and $b \in \mathbb{R}^{m}$ are vectors of parameters. Let us define the $\mu$-perturbed KKT system associated to the primal-dual pair (1) as

$$
\begin{aligned}
A x & =b \\
A^{T} y+s & =c \\
X S e & =\mu e, \\
(x, s) & >0,
\end{aligned}
$$

where $X=\operatorname{diag}\left(x_{1}, x_{2}, \ldots, x_{n}\right), S=\operatorname{diag}\left(s_{1}, s_{2}, \ldots, s_{n}\right), e=(1,1, \ldots, 1)$ in appropriate dimension and $\mu$ is defined as the barrier parameter which defines the central path. Equations $(2 \mathrm{a})$ and $(2 \mathrm{~b})$ are the linear constraints corresponding to primal and dual feasibility, respectively, and equations (2c) are the perturbed complementarity conditions, which are (mildly) non-linear (bilinear term).

In a primal-dual interior point method, an approximate solution to the perturbed KKT system (2) is obtained at each iteration by using a (damped) step of a Newton-like system of equations. The step direction is defined by the vector $(\Delta x, \Delta y, \Delta s)$ which is obtained by solving the following system of equations

$$
\left[\begin{array}{ccc}
A & 0 & 0 \\
0 & A^{T} & I \\
S^{l} & 0 & X^{l}
\end{array}\right]\left[\begin{array}{c}
\Delta x^{l} \\
\Delta y^{l} \\
\Delta s^{l}
\end{array}\right]=\left[\begin{array}{c}
\xi_{b}^{l} \\
\xi_{c}^{l} \\
\tau \mu_{l} e-X^{l} S^{l} e
\end{array}\right],
$$

where the primal-dual solution at iteration $l$ is $(x, y, s)=\left(x^{l}, y^{l}, s^{l}\right)$, and $\xi_{b}^{l}=$ $b-A x^{l}$ and $\xi_{c}^{l}=c-A^{T} y^{l}-s^{l}$ are the primal and dual residuals at iteration $l$, respectively and $\tau \in(0,1)$ is the centering parameter.

Once direction $\left(\Delta x^{l}, \Delta y^{l}, \Delta s^{l}\right)$ is determined from (3) and since the next iterate is defined as $\left(x^{l+1}, y^{l+1}, s^{l+1}\right)=\left(x^{l}+\alpha_{P} \Delta x^{l}, y^{l}+\alpha_{D} \Delta y^{l}, s^{l}+\alpha_{D} \Delta s^{l}\right)$ suitable values for $\alpha_{P} \in[0,1]$ and $\alpha_{D} \in[0,1]$ are calculated bearing in mind 
that $\left(x^{l+1}, s^{l+1}\right)>0$. After this, $\mu_{l+1}$ is updated (usually reduced) and the primal-dual method continues iterating until a relative duality gap drops below a prescribed optimality tolerance $\varepsilon$. The stopping criterion is usually defined as

$$
\frac{c^{T} x-b^{T} y}{1+\left|c^{T} x\right|} \leq \varepsilon .
$$

One of the most remarkable characteristics of primal-dual methods is that the distance to optimality, called the duality gap, can be calculated if some conditions hold. The analytic $\mu$-centre, $(x(\mu), y(\mu), s(\mu))$, is defined as the unique point satisfying conditions (2a)-(2c) and $(x(\mu), s(\mu))>0$ for a particular $\mu>0$. The duality gap can be calculated as

$$
c^{T} x-b^{T} y=c^{T} x-(A x)^{T} y=x^{T}\left(c-A^{T} y\right)=x^{T} s=n \mu .
$$

\section{Column generation}

In this section we briefly describe the ideas behind column generation. For a more detailed explanation of the method, see [25]. The aim of a column generation method is to solve a problem called the master problem (MP) through a restricted version of it (e.g., a problem with fewer variables). Initial columns are chosen to construct the restricted master problem (RMP) usually via a heuristic procedure, a known feasible solution or using artificial columns. Then, the RMP is solved and dual solutions of the RMP are used in the oracle. The oracle, also known as the subproblem, is a problem which usually hides the complexity of the original problem (i.e., integrality). After solving the oracle new columns with negative reduced costs are obtained and added to the RMP, if any. This process continues until the stopping criterion is satisfied. The solution of the last RMP provides the optimal solution of the MP. Note that from one iteration to another we would like to take advantage of the solution process of the previous RMP in order to speed up the solution process of the new RMP after adding new columns.

Column generation is widely applied in the context of mixed-integer programming. In this paper we analyse this method after relaxing a mixed-integer programming problem using Dantzig-Wolfe decomposition (DWD). Loosely speaking, DWD aims to represent the feasible set of an integer problem using its convex hull and optimizes over this set. This convex hull is described by a linear convex combination of extreme points and extreme rays. We are interested in the case when the instances have a compact non-empty feasible set and therefore, only extreme points are needed to describe the convex hull of the original problem. Also, we consider a relaxation of the integer variables of the original problem (i.e., root problem in a branch-and-bound context) and thus, we have a sequence of linear optimization problems to solve. Since the number of extreme points is likely to be large and we only need to describe the convex hull in the region close to the optimal solution, one should rely on methods that exploit this fact. Column generation is such method. 
Let us assume that we are in an intermediate iteration of the column generation process. The current RMP (1) characterized by $A, b, c$ has been solved and the oracle generates $k$ new columns with parameters $(\bar{A}, \bar{c})$. Note that $\bar{c}_{j}-\bar{A}_{j}^{T} y<0$ for all $j \in K=\{1, \ldots, k\}$ for a dual solution $y$ of the current RMP. The modified primal-dual pair is

$$
\begin{aligned}
& \mathcal{P}_{1}:=\min \quad c^{T} x+\bar{c}^{T} \bar{x}, \quad \text { s.t. } \quad A x+\bar{A} \bar{x}=b, \quad x \geq 0, \quad \bar{x} \geq 0, \\
& \mathcal{D}_{1}:=\max \quad b^{T} y, \quad \text { s.t. } \quad A^{T} y+s=c, \quad \bar{A}^{T} y+\bar{s}=\bar{c}, \quad s \geq 0, \quad \bar{s} \geq 0,
\end{aligned}
$$

where $k$ is the number of columns (variables) added to the original problem (1), $\bar{x} \in \mathbb{R}^{k}$ is the vector of new primal variables and $\bar{s} \in \mathbb{R}^{k}$ is the vector of new dual slack variables. $\bar{A} \in \mathbb{R}^{m \times k}$ represents the coefficient matrix for the new variables and $\bar{c} \in \mathbb{R}^{k}$ the vector of objective function coefficients. Note that new columns in the primal space are associated to new constraints in the dual space. Constraints in the dual space can be interpreted as the cuts which restrict the dual localization set. From now on we will use both, columns and cuts where the former term refers to the primal space while the latter to the dual. After dropping the iteration index $l$ and similar to (3), the new Newton-like system of equations is

$$
\left[\begin{array}{ccccc}
A & \bar{A} & 0 & 0 & 0 \\
0 & 0 & A^{T} & I & 0 \\
0 & 0 & \bar{A}^{T} & 0 & I \\
S & 0 & 0 & X & 0 \\
0 & \bar{S} & 0 & 0 & \bar{X}
\end{array}\right]\left[\begin{array}{c}
\Delta x \\
\Delta \bar{x} \\
\Delta y \\
\Delta s \\
\Delta \bar{s}
\end{array}\right]=\left[\begin{array}{c}
\xi_{\bar{b}} \\
\xi_{c} \\
\xi_{\bar{c}} \\
\tau \mu e-X S e \\
\tau \mu e-\bar{X} \bar{S} e
\end{array}\right]
$$

where $\xi_{\bar{b}}=b-A x-\bar{A} \bar{x}, \xi_{\bar{c}}=\bar{c}-\bar{A}^{T} y-\bar{s}, \bar{X}=\operatorname{diag}\left\{\bar{x}_{1}, \ldots, \bar{x}_{k}\right\}, \bar{S}=$ $\operatorname{diag}\left\{\bar{s}_{1}, \ldots, \bar{s}_{k}\right\}, \mu=\left(x^{T} s+\bar{x}^{T} \bar{s}\right) /(n+k)$ and $\tau \in(0,1)$ is the centering parameter.

The primal-dual method under which we design our warmstarting strategy is the one described in [15]. This technique relies on well-centred close-tooptimal solutions of the RMPs which are obtained by a primal-dual interior point method. This method takes into account that solving the RMP to optimality is not needed in the early stages of the column generation procedure as it was also pointed out in $[26,29]$. Loosely speaking, a RMP has to be solved to optimality only at the last iteration of the column generation. An important feature of this method is that even that in one iteration the algorithm may not provide any new column, it guarantees the progress by decreasing the required distance to optimality. Termination is achieved when there are no more columns to append and the relative gap drops below a prescribed optimality tolerance. The method has shown encouraging results in solving the root node of several combinatorial optimization problems [15]. Despite the fact the warmstarting strategy presented in this paper was proposed to improve the performance in this specific context, it could be easily extended to any situation where a linear optimization problem is solved using a column generation or cutting plane framework with a primal-dual interior point method. 


\section{Warmstarting strategy}

In this paper we introduce a specialized warmstarting strategy applicable within the primal-dual column generation scheme [15]. Specialized because we take advantage of some structure/properties which are often present when solving combinatorial optimization problems. We have observed that in many applications all the elements in $A$ and $\bar{A}$ are non-negative. For instance, columns in the primal space describing cutting patterns (cutting stock problem [10]), routes (vehicle routing problem [22]) or production plans (lot sizing problem [35]) by definition contain only non-negative entries. In our developments we do consider this observation and therefore, the analysis and results should be understood in this context. For a description of each of the restricted master problems obtained after applying a Dantzig-Wolfe reformulation [5] for each of these applications, we refer the reader to [15] and the references therein.

The process of finding a warmstarting point has been divided into two stages. In the first, we need to find a point from a list of stored iterates which satisfies some properties. In the other, an adjustment $(\Delta x, \Delta y, \Delta s)$ has to be computed taking this point as a reference. After this, the step is taken in this direction and the old point is updated in the new dimensions to produce a full-dimension warmstarting point. From this point we continue iterating and solving system (6). Our aim is to find a starting point which reduces the number of iterations required to solve problem (5) when compared to a coldstart approach. To develop such a two-stage approach we need to deal with two problems: (a) feasibility in the primal and dual spaces and (b) centrality of the new warmstarting point. We address both issues later.

Since we are designing a new warmstarting technique to be applied in the PDCGM, it is sensible to point out the similarities and main differences with the strategy currently in use [14] and other strategies presented in the literature such as the ones studied in [37] and [12].

Despite the fact that our developments follow the idea of Gondzio in [14] of treating primal and dual infeasibilities separately, our approach differs in two key aspects. Firstly, in the choice of $\bar{x}$ and $\bar{s}$. The aforementioned paper sets $\bar{s}_{j}=\max \left\{\left|\bar{c}_{j}-\bar{A}_{j}^{T} y\right|, \mu^{1 / 2}\right\}$, for every added cut $j$. Since it is likely to have (deep) cuts where $\bar{c}_{j}-\bar{A}_{j}^{T} y \ll 0, \bar{s}_{j}$ usually takes large values. Furthermore, since the complementarity products of the new variables are set to $\bar{x}_{j} \bar{s}_{j}=\mu$, for every $j=1,2, \ldots, k$, such strategy is likely to produce small values of $\bar{x}_{j}$. This goes against the expectations that the new variables corresponding to the recently appended (deep) cuts are likely to take non-zero values at the optimal solution. In our strategy we do not choose $\bar{x}_{j}$ as a function of $\bar{s}_{j}$. Instead, we choose these values bearing in mind their impact on centrality.

A second difference between the strategy of [14] and ours is in the definition of the search direction to find the new warmstarting point. In the method proposed in [14], the search direction is calculated using a variation of system (6) via target $t$, which is a consequence of the choice of $\bar{x}_{j}$ and $\bar{s}_{j}$. In the primal space, feasibility is restored by choosing small $\bar{x}_{j}$ 's. In the dual space, the method ensures that after a full step is taken in this direction, dual feasibility 
is recovered in the new components. However, this method does not guarantee the same for the old components. It is accepted that the complementarity products for the old variables may get significantly worse as a price to pay for recovering primal and dual feasibilities. In our proposed strategy, we aim to restore primal and dual feasibilities using auxiliary linear optimization problems. The principal aim of both, primal and dual auxiliary problems, is to seek feasibility while minimizing changes in old variables which are small in order to avoid large changes in the complementarity products. Our approach follows a variation of a weighted least-square strategy proposed in [37]. It differs from [37] in the sense that we only consider small variables and that we have extra constraints to satisfy in both, primal and dual spaces.

The method presented in this paper has several other different characteristics when compared with the strategy proposed in [12]. Firstly, in [12,31], the strategy recovers primal and dual feasibility by solving a non-linear problem while in our approach we aim to minimize large variations in the old components which are small using linear functions and extra constraints. Secondly, the strategies proposed in $[12,31]$ work very well if all the new constraints traverse the Dikin's ellipsoid around the query point while our approach relies on a different assumption which is that the depth of the added constraints does not exceed a reference value which is a function of $\mu$ and $\gamma$, where $\gamma$ defines how close we keep the complementary products with respect to the barrier parameter (see our definition $\mathcal{N}_{s}(\gamma)$ below). Thirdly, we are able to retreat back in the list of iterates while the strategies in $[12,31]$ take the last iterate as the reference point and adjust it. In spirit, the strategies developed for the analytic centre cutting plane method $[12,31]$ and the one described in this paper aim to the same objective which is to obtain a well-centred warmstarted iterate after adding new columns/constraints, however while our approach relies on the notion of the central path, the other approaches aim to get a point close to the new analytic centre.

In what follows of this Section, we analyse restoring primal and dual feasibilities, the consequences in the new complementarity products and describe the complete algorithm.

Let $\left(x^{0}, y^{0}, s^{0}\right)$ be a feasible solution of the primal-dual pair (1). Also, let us define the primal-dual strictly feasible set and the central path neighbourhood as follows

$$
\begin{aligned}
& \mathcal{F}^{0}=\left\{(x, y, s): A x=b ; A^{T} y+s=c ;(x, s)>0\right\}, \\
& \mathcal{N}_{s}(\gamma)=\left\{(x, y, s) \in \mathcal{F}^{0}: \gamma \mu \leq x_{j} s_{j} \leq \frac{1}{\gamma} \mu ; \forall j=1,2, \ldots, n\right\} .
\end{aligned}
$$

We are interested in $\left(x^{0}, y^{0}, s^{0}\right) \in \mathcal{N}_{s}(\gamma)$ for a fixed $\gamma \in(0,1)$. The reader must note that our definition of the neighbourhood is a slight modification of the wide neighbourhood $\mathcal{N}_{-\infty}(\gamma)$ in [36] proposed in [16]. The aim of our specialized warmstarting algorithm is to find an initial point from where to start solving the modified problem (5). This initial point is obtained from information gathered when solving problem (1) via system of equations (3) with $\xi_{b}=\xi_{c}=0$. 
Before continuing, let $N=\{1, \ldots, n\}, M=\{1, \ldots, m\}$ and $K=\{1, \ldots, k\}$ be index sets. Additionally, let us state the following general assumptions.

(A.1) All elements in matrices $A$ and $\bar{A}$ are non-negative.

(A.2) We are able to store a list of iterates which are strictly feasible and well-centred (i.e., $\left(x^{0}, y^{0}, s^{0}\right) \in \mathcal{N}_{s}(\gamma)$ ).

(A.3) There exists a $\mathcal{U}_{v}$ such as $\left\|x^{0}, s^{0}\right\|_{\infty} \leq \mathcal{U}_{v}$.

(A.4) $\mathcal{U}_{v}^{2}>1$.

Assumption (A.1) is motivated by the applications we are interested in solving. There are several examples in combinatorial optimization where this assumption is valid. We refer the reader to [15] to see three applications in which this assumption holds. Additionally, there is a wide variety of problems which satisfy this condition. See for instance the travelling salesman problem description in [2] where the authors consider the Held and Karp 1-tree relaxation $[19,20]$ or the uncapacitated facility location problem [4] just to mention some of them.

For the clarity of the exposition, we have explicitly state Assumption (A.1), although it can be weakened by adding a preprocessing stage in the following way. Considering Assumption (A.2) implies (in a more general way) that the variables are bounded. Let us consider that $\|x\|_{\infty} \leq \mathcal{U}_{x}$, where $\mathcal{U}_{x}>0$. Additionally, let us introduce a non-negative variable $\hat{x} \in \mathbb{R}_{+}^{n}$ such that $x_{j}+\hat{x}_{j}=\mathcal{U}_{x}$, for every $j \in N$. Additionally, and for every $i \in M$, let us create two disjoint sets such that $N_{i}^{+}$contains indices $j$ 's for which $a_{i j} \geq 0$. Similarly, $N_{i}^{-}$contains indices $j$ 's for which $a_{i j}<0$. For every $i \in M$, we have that $N_{i}^{+} \cup N_{i}^{-}=N$. Using $x_{j}=\mathcal{U}_{x}-\hat{x}_{j}$ and without loss of generality, we can rewrite $A x=b$ as

$$
\sum_{j \in N_{i}^{+}} a_{i j} x_{j}+\sum_{j \in N_{i}^{-}} \hat{a}_{i j} \hat{x}_{j}=b_{i}+\sum_{j \in N_{i}^{-}} \hat{a}_{i j} \mathcal{U}_{x}, \quad \forall i \in M,
$$

where $\hat{a}_{i j}:=-a_{i j}>0$. Hence, we now have only nonnegative coefficients. Although this preprocessing stage guarantees that Assumption (A.1) is satisfied (so we can safely remove it), it requires the addition of more constraints and variables and therefore its benefits in practice has yet to be evaluated.

Assumption (A.2) is easily met if the feasible path following algorithm is used to solve the problem.

Assumption (A.3) is a technical requirement similar to the one used in [37] which bounds the largest primal and dual slack variables. Note that in [37, Theorem 2.1.] and following the analysis in [30], the authors have shown that for a strictly feasible point $(x, y, s)$ which does not necessarily lie exactly on the central path but it belongs to its neighbourhood, the following bounds are satisfied

$$
\|x\|_{2} \leq \mathcal{C}(d)\left(\mathcal{C}(d)+\frac{\mu n}{\|d\|_{2}}\right) \text { and }\|s\|_{2} \leq 2\|d\|_{2} \mathcal{C}(d)\left(\mathcal{C}(d)+\frac{\mu n}{\|d\|_{2}}\right),
$$

where $d$ represents a triplet containing the instance data $(d=(A, b, c)), \mathcal{C}(d)$ is the condition number [30] and recalling $\mu=x^{T} s / n$. Similarly, in our case it is 
easy to see that $\mathcal{C}(d)<\infty$ (feasible RMPs) and since we use a path-following method and a minor variation of the neighbourhoods used in [37], Assumption (A.3) is satisfied.

Assumption (A.4) is a also a technical condition which can be easily imposed by setting $\mathcal{U}_{v}=\max \left\{\left\|x^{0}, s^{0}\right\|_{\infty} ; 1+\epsilon\right\}$, where $\epsilon=10^{-8}$.

Additionally, to these assumptions and due to the nature of our applications, we can always initialize the column generation procedure with some initial columns so the initial RMP is feasible (see [15] and reference therein).

Using our definition of the central path neighbourhood for $\left(x^{0}, y^{0}, s^{0}\right) \in$ $\mathcal{N}_{s}(\gamma)$, we have

$$
\gamma \mu_{0} \leq x_{j}^{0} s_{j}^{0} \leq \frac{1}{\gamma} \mu_{0} \quad \forall j \in N .
$$

Both, this definition and the upper bound $\mathcal{U}_{v}$, allow us to bound $x_{j}^{0}$ and $s_{j}^{0}$, for every $j \in N$, as follows

$$
\begin{aligned}
& \frac{\gamma \mu_{0}}{\mathcal{U}_{v}} \leq x_{j}^{0} \leq \mathcal{U}_{v}, \\
& \frac{\gamma \mu_{0}}{\mathcal{U}_{v}} \leq s_{j}^{0} \leq \mathcal{U}_{v} .
\end{aligned}
$$

The similarities between the initial RMP and the modified RMP are one of the key elements to take into consideration when a warmstarting strategy is designed for an interior point method. If there is no similarity between the initial and modified problems, we can expect that a warmstarting strategy will not lead to any substantial improvement when compared with a coldstart approach. This could be the case when the new RMP has a completely different feasible region around the optimal solution and a close-to-optimality solution of the previous problem deeply violates the new constraints. Therefore, any information previously gathered close to the optimal solution will not help to speed up the solution process of the modified problem. Then, there is a need to understand the relation between the added cuts and the previous RMP. A sensible way to proceed is to measure the size of the newly added cuts in terms of the current penalty parameter, $\mu_{0}$. Let the inequality $-\left(\bar{c}_{j}-\bar{A}_{j}{ }^{T} y^{0}\right) \leq f\left(\mu_{0}\right)$ be used to determine the depth of the cuts, where $f\left(\mu_{0}\right)$ is an increasing function of $\mu_{0}$. Note that by using this idea of associating the depth of the cuts to $\mu_{0}$ and having a list of iterates, we could retreat far enough in the iteration process to make the cuts relatively shallower. In other words, for all the cuts we could choose a suitably large $\mu_{0}$ to decrease their relative depth.

As mentioned earlier, the choices of $\bar{x}$ and $\bar{s}$ have important consequences in the primal-dual infeasibilities and in the complementarity conditions. We aim to find a warmstart which: (a) is feasible in the primal and dual space; and (b) keeps the complementarity products reasonably small and inside a slightly modified neighbourhood if the cuts satisfy some desirable properties. We expect the duality gap to increase since we are adding variables/constraints to the primal/dual problem. However, we would like to keep this duality measure relatively close to the old one.

Now, let us define some sets and parameters used throughout the paper. 
Definition 1 Let $\mathcal{B}_{0}$ be the set containing all indices $j$ such that $x_{j}^{0} \geq s_{j}^{0}$, where $j \in N$. We call this set the primal dominant partition at solution $\left(x^{0}, y^{0}, s^{0}\right)$. Conversely, the dual dominant partition at solution $\left(x^{0}, y^{0}, s^{0}\right)$ is defined as $\mathcal{N}_{0}$ and contains all indices $j$ such that $x_{j}^{0}<s_{j}^{0}$, where $j \in N$.

The reader familiar with the simplex method may be tempted to interpret the sets $\mathcal{B}_{0}$ and $\mathcal{N}_{0}$ as a guess of the basic-nonbasic partition. In the spirit of IPM, these sets provide only an early guess of the primal-dual strictly complementarity partition which in general is not equivalent to the basicnonbasic partition.

Definition 2 Considering $A \in \mathbb{R}_{+}^{m \times n}$, let us define

$$
A_{\min }:=\min _{i \in M, j \in N: A_{i j}>0}\left\{A_{i j}\right\},
$$

as the minimum non-zero element of matrix $A$. Similarly,

$$
a_{\min }:=\min _{i \in M: a_{i}>0}\left\{a_{i}\right\}
$$

is the minimum non-zero element of vector $a \in \mathbb{R}_{+}^{m}$. Additionally, $\sigma_{\max }(A)$ and $\sigma_{\min }(A)$ denote the maximum and minimum singular value of matrix $A$, respectively and

$$
\sigma_{\max }=\max \left\{\sigma_{\max }(A), \sigma_{\max }(\bar{A})\right\}
$$

In the following three sections we will prove the main results of this paper. Namely, we will provide the methodology to choose a good warmstart solution such that the primal and dual feasibilities may be restored in one Newton step and will show (in Section 4.3) that this can be achieved without significantly affecting the proximity of the new iterate to the central path.

\subsection{Dual feasibility}

In order to minimize the impact of restoring dual feasibility and to measure its effect on complementarity products, we have defined an auxiliary linear optimization problem. Taking the second and third equations of (6) and considering $\left(x^{0}, y^{0}, s^{0}\right) \in \mathcal{F}^{0}$, we have

$$
\begin{aligned}
A^{T} \Delta y+\Delta s & =0, \\
\bar{A}^{T} \Delta y+\Delta \bar{s} & =\bar{c}-\bar{A}^{T} y^{0}-\bar{s} .
\end{aligned}
$$

Such a formulation allows for a considerable freedom in the choice of $\Delta y$. This system of equations is likely to have multiple solutions.

The following auxiliary linear optimization problem minimizes the relative change of variables in the primal dominant partition (i.e., corresponding to small $s_{j}^{0}$ ) when a solution of problem (1) is available. 


$$
\begin{aligned}
& \min _{\Delta y, \Delta s, \Delta \bar{s}} \sum_{j \in \mathcal{B}_{0}} \frac{\Delta s_{j}}{s_{j}^{0}}, \\
& \text { s.t. } \quad \sum_{i \in M} \bar{A}_{i j} \Delta y_{i}+\Delta \bar{s}_{j}=\bar{c}_{j}-\sum_{i \in M} \bar{A}_{i j} y_{i}^{0}-\bar{s}_{j}, \quad \forall j \in K, \\
& \text { - } \sum_{i \in M} A_{i j} \Delta y_{i}=\Delta s_{j}, \quad \forall j \in \mathcal{B}_{0}, \\
& \Delta y_{i} \leq 0, \\
& \forall i \in M, \\
& \Delta \bar{s}_{j} \geq 0,
\end{aligned}
$$

Observe that by solving this linear problem, feasibility for the new components is achieved via constraints (11b) (the third equation of system (6)). Note that the aim of this linear program is to minimize the relative change of small slacks of the dual problem while a full step is feasible for all components. The motivation behind this objective is that we would like to perturb the previous solution as little as possible. By setting

$$
\bar{s}_{j}=\max \left\{\gamma \mu_{0} \frac{\bar{A}_{\min }}{\sqrt{m} \mathcal{U}_{v} \sigma_{\max }}, \bar{c}_{j}-\bar{A}_{j}^{T} y^{0}\right\}, \quad \forall j \in K,
$$

and noting that $\bar{s}_{j}^{w}=\bar{s}_{j}+\Delta \bar{s}_{j}$ (new slack variables) for every $j \in K$, constraints (11e) ensure that the new slack variables are bounded away from zero. Although one would expect to have

$$
\gamma \mu_{0} \frac{\bar{A}_{\min }}{\sqrt{m} \mathcal{U}_{v} \sigma_{\max }}>0>\bar{c}_{j}-\bar{A}_{j}^{T} y^{0}, \quad \forall j \in K,
$$

this is not always the case due to the backtracking feature of our strategy. This will be explained in more detail later in the paper.

The linear problem (11) can be simplified by the following steps. First, let us eliminate $\Delta s_{j}$ for every $j \in \mathcal{B}_{0}$ by using constraints (11c) and substitute $\Delta y=-\Delta \bar{y}$. Also, let us introduce a new parameter $f_{i}$ for every $i \in M$ such that $f_{i}=\sum_{j \in \mathcal{B}_{0}} \frac{A_{i j}}{s_{j}^{0}}$. Now, we can rewrite problem (11) as

$$
\begin{aligned}
& \mathcal{D}_{\text {aux }}:=\min _{\Delta \bar{y}, \Delta \bar{s}} \sum_{i \in M} f_{i} \Delta \bar{y}_{i} \\
& \text { s.t. } \sum_{i \in M} \bar{A}_{i j} \Delta \bar{y}_{i}-\Delta \bar{s}_{j}=-\left(\bar{c}_{j}-\sum_{i \in M} \bar{A}_{i j} y_{i}^{0}-\bar{s}_{j}\right), \quad \forall j \in K, \\
& \Delta \bar{y}_{i} \geq 0, \\
& \Delta \bar{s}_{j} \geq 0,
\end{aligned}
$$

To avoid unbounded solutions, if $f_{i}=0$, for a given $i \in M$, we set $f_{i}=1$. Observe then that by assumption (A.1), $f_{i}>0$ for every $i \in M$. Therefore, the problem (13) can be interpreted as finding the minimum adjustment $\Delta \bar{y}$ 
of dual variables $y$ such that at the new point $y+\Delta y(=y-\Delta \bar{y})$ all dual feasibility constraints in (5b), including the ones corresponding to new deep cuts, are satisfied. The objective function (13a) promotes such changes $\Delta \bar{y}$ which do not alter the primal dominant components of $s^{0}$ (the components corresponding to small values $s_{j}^{0}$ ).

Let us introduce $d \in \mathbb{R}_{+}^{k}$ such that

$$
\bar{A}^{T} \Delta \bar{y}-\Delta \bar{s}=-\left(\bar{c}-\bar{A}^{T} y^{0}-\bar{s}\right)=d .
$$

If $\bar{c}_{j}-\bar{A}_{j}^{T} y^{0}<\gamma \mu_{0} \frac{\bar{A}_{\min }}{\sqrt{m} \mathcal{U}_{v} \sigma_{\max }}$ and from definition (12), clearly $d_{j}>0$. When $\bar{c}_{j}-\bar{A}_{j}^{T} y^{0} \geq \gamma \mu_{0} \frac{\bar{A}_{\min }}{\sqrt{m} \mathcal{U}_{v} \sigma_{\max }}$, the corresponding $d_{j}=0$.

The purpose of the next lemma is to show that the problem $\mathcal{D}_{\text {aux }}$ has a bounded solution.

Lemma 1 If Assumption (A.1) holds and given $\bar{c}_{j}-\bar{A}_{j}^{T} y^{0}$ for every $j \in K$ representing the reduced cost of column $j$, where $\bar{A}_{j}$ represents the $j$-th column of matrix $\bar{A}$, problem (13) has a bounded solution.

Proof Since $f>0$ and $\Delta \bar{y} \geq 0$, it follows that $\Delta \bar{y}$ is bounded from both sides. Moreover, $\Delta \bar{s} \geq 0$ so problem (13) has a bounded solution.

It is not hard to prove that a valid bound of $\Delta \bar{y}$ is

$$
\Delta \bar{y}_{i} \leq \max _{j \in K}\left\{\Upsilon_{j}\right\}, \forall i \in M
$$

where $\Upsilon_{j}=d_{j} /\left(\bar{A}_{j}\right)_{\text {min }}$, for every $j \in K$.

If $d_{j}=0$ for every $j \in K$, the trivial solution $(\Delta \bar{y}, \Delta \bar{s})=0$ is the optimal solution. This case is unlikely since we expect columns with negative reduced costs, namely $\bar{c}_{j}-\bar{A}_{j}^{T} y^{0}<0$. However, and as it will be explained later, due to our strategy in some occasions this may happen.

The next lemma states that by calculating direction $\Delta \bar{y}$ from (13) infeasibilities are absorbed in the old components and a dual feasible point for the new problem is obtained.

Lemma 2 Let $\left(x^{0}, y^{0}, s^{0}\right) \in \mathcal{F}^{0}$. If $\Delta \bar{y}$ is chosen by solving (13) then a full step in the old components is feasible. Therefore, dual feasibility is restored in one step.

Proof From the definition of problem (13), we have $\Delta \overline{y_{i}} \geq 0$ for every $i \in M$ and therefore, $\Delta y_{i} \leq 0$ for every $i \in M$. Since $A_{i j} \geq 0$ for every $j \in N$ and $i \in M$ and using (9), we have:

$$
\Delta s_{j}=-\sum_{i \in M} A_{i j} \Delta y_{i} \geq 0, \quad \forall j \in N .
$$

Recalling that $s_{j}^{0}>0, s_{j}^{0}+\Delta s_{j}>0, \forall j \in N$ as required. 
Note that Lemma 2 only requires a feasible $\Delta \bar{y}$ and $\Delta \bar{s}$ for problem (13) and we do not need to solve (13) to optimality. However, we believe that our objective function in (13) promotes small perturbations in the complementarity products of the warmstarting iterate and therefore, we also seek optimality.

Even though our objective is to minimize the variation of the dual elements in the primal dominant partition, we cannot guarantee this variation to be small. This is an unavoidable consequence of the fact that there is no control of the depth of the new cuts and a large variation of some components $\Delta s$ is expected. Despite the lack of control, we can still determine an upper bound for $\Delta s$ in the old components. Note that from (9) and $\Delta y=-\Delta \bar{y}$, we have

$$
\Delta s=A^{T} \Delta \bar{y},
$$

Also, from (15) we have the following bound on $\Delta \bar{y}_{i}$ for every $i \in M$

$$
\Delta \bar{y}_{i} \leq \frac{d_{\max }}{\bar{A}_{\min }}
$$

where, following (14) and(15), $d_{\max }$ is defined as

$$
d_{\text {max }}=\max _{j \in K}\left\{-\bar{c}_{j}+\sum_{i \in M} \bar{A}_{i j} y_{i}^{0}+\bar{s}_{j}\right\} .
$$

Applying $l_{\infty}$ norm to (16) and considering the upper bound of (17), we obtain

$$
\begin{aligned}
\|\Delta s\|_{\infty} & \leq\left\|A^{T}\right\|_{\infty}\|\Delta \bar{y}\|_{\infty} . \\
& \leq \frac{\sqrt{m} \sigma_{\max }(A) d_{\max }}{\bar{A}_{\min }} .
\end{aligned}
$$

Thus,

$$
\Delta s_{j} \leq \frac{\sqrt{m} \sigma_{\max } d_{\max }}{\bar{A}_{\min }}, \quad \forall j \in N .
$$

Additionally, considering (14), for every $j \in K$, we have

$$
\bar{s}_{j}+\Delta \bar{s}_{j} \leq \frac{\sqrt{m} \sigma_{\max } d_{\max }}{\bar{A}_{\min }}+\left(\bar{c}_{j}-\bar{A}_{j}^{T} y^{0}\right), \quad \forall j \in K .
$$

\subsection{Primal feasibility}

Similarly to recovering dual feasibility, we aim to restore feasibility in the primal space by solving an auxiliary linear optimization problem and using the notion of primal dominant and dual dominant partitions. Considering the first equation of system $(6)$ and $\left(x^{0}, y^{0}, s^{0}\right) \in \mathcal{F}^{0}$, we have

$$
A \Delta x=-\bar{A}(\bar{x}+\Delta \bar{x})
$$

To simplify the notation, let us define $\bar{x}_{j}^{w}=\bar{x}_{j}+\Delta \bar{x}_{j}$ for every $j \in K$. Primal feasibility would be easily restored if we could set $\bar{x}^{w}=0$. Since in 
interior point methods this is not possible, we need to fix or determine a positive value for $\bar{x}^{w}$. In practice, primal feasibility could still be easily achieved by setting $\bar{x}^{w}$ sufficiently small. We could apply for example the primal feasibility restoration direction proposed in [14], $\Delta x=-\Theta A^{T}\left(A \Theta A^{T}\right)^{-1} \bar{A} \bar{x}^{w}$ for non-degenerate systems where $\Theta=X S^{-1}$ is a diagonal scaling matrix. This is a generalization of Mitchell's direction presented in [27] and applied in the primal projective algorithm to handle multiple cuts. Setting a small value for $\bar{x}^{w}$ seems to be sensible since primal infeasibility depends on this value. We have designed a slightly different strategy which takes into account setting $x_{j}^{0}$ small but also considering centrality aspects. For now, it is enough to say that our choice of $\bar{x}^{w}$ is the maximum possible value in order to ensure that: (a) a full step in the primal direction is possible, and (b) primal feasibility is restored. Similar to the dual feasibility restoration, we have defined the following auxiliary linear optimization problem.

$$
\begin{aligned}
& \mathcal{P}_{\text {aux }}:=\min _{\Delta x, \Delta x^{+}, \Delta x^{-}} \sum_{j \in \mathcal{N}_{0}} \frac{\Delta x_{j}^{+}+\Delta x_{j}^{-}}{x_{j}^{0}}, \\
& \text { s.t. } \sum_{j \in N} A_{i j} \Delta x_{j}=-\sum_{j \in K} \bar{A}_{i j} \bar{x}_{j}{ }^{w}, \quad \forall i \in M \text {, } \\
& \Delta x_{j} \geq x_{j}^{0}\left(\delta_{l}-1\right), \quad \forall j \in N, \\
& \Delta x_{j}=\Delta x_{j}^{+}-\Delta x_{j}^{-}, \quad \forall j \in \mathcal{N}_{0}, \\
& \Delta x_{j}^{+} \geq 0, \quad \forall j \in \mathcal{N}_{0}, \\
& \Delta x_{j}^{-} \geq 0, \quad \forall j \in \mathcal{N}_{0},
\end{aligned}
$$

where $\delta_{l}$ is a given parameter which satisfies $0<\delta_{l}<1$ and its meaning will be explained later in the paper. In the primal case and similar to what we did for the dual variables in the primal dominant partition, we minimize the relative change of the variables in the dual dominant partition (corresponding to small $x_{j}^{0}$ ). Note that in this case we allow positive and negative directions and therefore we minimize the absolute value of such directions. Constraints (21b) guarantee that by taking a full step in direction $\Delta x$, feasibility in the primal space is completely restored. Constraints (21c) ensure that if we take a full step in direction $\Delta x$, the new iterate will remain positive. Constraints (21d)-(21f) are additional requirements that help to calculate the absolute value of every $\Delta x_{j}$ in the dual dominant partition. If $\Delta x_{j}>0$, then $\Delta x_{j}^{+}>0$ and $\Delta x_{j}^{-}=0$. If $\Delta x_{j}<0$, then $\Delta x_{j}^{-}>0$ and $\Delta x_{j}^{+}=0$. Finally, if $\Delta x_{j}=0$, then both $\Delta x_{j}^{+}$and $\Delta x_{j}^{-}$are zero. Note that ensuring a non-empty feasible set in $\mathcal{P}_{\text {aux }}$ for general $A$ and $\bar{A}$ is a non-trivial task. For instance, large values on $\bar{x}^{w}$ may lead to large negative values in some $\Delta x_{j}$ and therefore, satisfying constraint (21c) may not be possible. Later we will define conditions to ensure that primal infeasibility is completely absorbed in one step. 
Following [37], let us use the following $Q R$ factorization of $A^{T}$,

$$
A^{T}=Q\left[\begin{array}{c}
R \\
0
\end{array}\right]=\left[Q_{1}, Q_{2}\right]\left[\begin{array}{c}
R \\
0
\end{array}\right]=Q_{1} R,
$$

where $Q_{1}$ is an $n \times m$ matrix, $Q_{2}$ is an $n \times(n-m)$ matrix, $\left[Q_{1}, Q_{2}\right]$ is a matrix with orthogonal columns, and $R$ is an $m \times m$ upper triangular matrix. It is easy to check that for the aforementioned factorization and given $\bar{x}^{w}$

$$
\Delta x=-Q_{1} R^{-T} \bar{A} \bar{x}^{w},
$$

satisfies equation (20). If $\mathcal{N}_{0}=\emptyset$, problem (21) becomes a feasibility problem and it only requires to find a solution which solves (21b) and (21c). Such a solution can be obtained from (23) given suitable value of $\bar{x}_{j}^{w}$ for every $j \in K$, as we will show later. It is known as the minimum norm solution. However, this is not the only solution $\Delta x$ which satisfies (20). Applying $l_{2}$ norm to (23), we have

$$
\|\Delta x\| \leq\left\|Q_{1}\right\|\left\|R ^ { - T } \left|\left\|\mid \bar{A} \bar{x}^{w}\right\| .\right.\right.
$$

Since $\left\|Q_{1}\right\|=1,\left\|R^{-T}\right\|=\sigma_{m i n}(R)^{-1}$ and $\sigma_{\min }(R)=\sigma_{\min }(A)$, it follows that

$$
\begin{aligned}
\|\Delta x\| & \leq \frac{\left\|\bar{A} \bar{x}^{w}\right\|}{\sigma_{\min }(A)} . \\
& \leq \frac{\sqrt{k} \sigma_{\max }(\bar{A}) \bar{x}_{\max }^{w}}{\sigma_{\min }(A)} .
\end{aligned}
$$

From (24), and using our definition of $\sigma_{\max }$, we can derive loose upper and lower bounds for every $\Delta x_{j}$, with $j \in N$. These bounds are

$$
-\frac{\sqrt{k} \sigma_{\max } \bar{x}_{\max }^{w}}{\sigma_{\min }(A)} \leq \Delta x_{j} \leq \frac{\sqrt{k} \sigma_{\max } \bar{x}_{\max }^{w}}{\sigma_{\min }(A)},
$$

and problem (21) has a bounded solution.

Let us define the feasible set of $\Delta x$ associated to $\mathcal{P}_{\text {aux }}$ as $\mathcal{S}_{\mathcal{P}}=\{\Delta x$ : (21b) - (21c) are satisfied $\}$. In addition, let us define a closely related set $\mathcal{S}_{\mathcal{Q}}=\{\Delta x:(21 \mathrm{~b})$ are satisfied $\}$. Observe that $\Delta x$ from (23) satisfies (21b) hence $\Delta x \in \mathcal{S}_{\mathcal{Q}}$. However, it does not have to satisfy (21c) and therefore in general $\Delta x \notin \mathcal{S}_{\mathcal{P}}$. The next lemma states that given a particular choice of $\bar{x}^{w}$ and the correct choice of $\mu_{0}$, we can guarantee that $\Delta x \in \mathcal{S}_{\mathcal{P}}$.

Lemma 3 Let $\left(x^{0}, y^{0}, s^{0}\right) \in \mathcal{N}_{s}(\gamma)$ for $\gamma \in(0,1), A_{i j} \geq 0, \forall i \in M, \forall j \in N$, $\bar{A}_{i j} \geq 0, \forall i \in M, \forall j \in K$. If

$$
\bar{x}_{j}^{w} \leq \gamma\left(1-\delta_{l}\right) \frac{\sigma_{\min }(A)}{\sqrt{k} \mathcal{U}_{v} \sigma_{\max }} \min \left\{\mu_{0}, \frac{1}{\mu_{0}}\right\}, \quad \forall j \in K,
$$

and $0<\delta_{l}<1$, then constraint (21c) is satisfied. 
Proof To ensure this, we have to show that (21c) is satisfied for every $\Delta x_{j}$ from (25). Hence, we have to prove that

$$
\left(1-\delta_{l}\right) x_{j}^{0} \geq \frac{\sqrt{k} \sigma_{\max } \bar{x}_{\max }^{w}}{\sigma_{\min }(A)} .
$$

Since we need to ensure this for every $j \in N$, it suffices if we check that this inequality is satisfied for the smallest possible $x_{j}^{0}$. We know from (8a) that $x_{j}^{0} \geq\left(\gamma \mu_{0}\right) / \mathcal{U}_{v}, \forall j \in N$. Therefore, we need to verify that

$$
\left(1-\delta_{l}\right) \frac{\gamma \mu_{0}}{\mathcal{U}_{v}} \geq \frac{\sqrt{k} \sigma_{\max } \bar{x}_{\max }^{w}}{\sigma_{\min }(A)} .
$$

This condition is satisfied if we choose $\bar{x}_{j}^{w}$ for every $j \in K$ satisfying (26) and by noting that $\mu_{0} \geq \min \left\{\mu_{0}, 1 / \mu_{0}\right\}$.

Note that with our choice of $\bar{x}_{j}^{w}$ satisfying (26) for every $j \in K$, we can find a solution to problem (21). As in the dual case, we now guarantee that a full step can be taken in direction $\Delta x$.

Lemma 4 Let $\left(x^{0}, y^{0}, s^{0}\right) \in \mathcal{N}_{s}(\gamma)$ with $\gamma \in(0,1)$ and $0<\delta_{l}<1$. By choosing $\Delta x$ from (21) and setting

$$
\bar{x}_{j}^{w}=\gamma\left(1-\delta_{l}\right) \frac{\sigma_{\min }(A)}{\sqrt{k} \mathcal{U}_{v} \sigma_{\max }} \min \left\{\mu_{0}, \frac{1}{\mu_{0}}\right\}, \quad \forall j \in K,
$$

we have $x_{j}^{0}+\Delta x_{j}>0$ and a full step in the primal space is feasible. Therefore, primal feasibility is restored in one step.

Proof Similarly to Lemma 2, from constraints (21c) and since $x_{j}^{0}>0$ and $0<\delta_{l}<1$, we deduce $x_{j}^{0}+\Delta x_{j}>0$ as required. Since this solution satisfies condition $(21 b)$, we conclude that the warmstart is primal feasible.

Lemma 4 only requires a feasible $\Delta x$ from problem (21). Similarly to the dual case, we also seek optimality in order to perturb the complementarity products as little as possible.

Additionally, using the bounds in (25), the choice of $\bar{x}^{w}$ in (27) and since $0<\delta_{l}<1$, we can guarantee

$$
-\left(1-\delta_{l}\right) \leq \frac{\Delta x_{j}}{x_{j}^{0}} \leq\left(1-\delta_{l}\right) .
$$

Note that our choice of $\bar{x}_{j}^{w}$ is independent of $\bar{s}_{j}^{w}$ for every $j \in K$. Moreover, and as described in Section $4.1, \bar{s}_{j}^{w}$ for every $j \in K$ is not fixed and must be calculated. However, and as we will see in the next section, both values are related via other parameters (i.e., $\mu_{0}$ and $\gamma$ ) in order to guarantee that complementarity products are still inside a slightly modified neighbourhood and that the new duality gap is also bounded.

Summarizing our findings so far, we have shown that by the use of $\mathcal{P}_{\text {aux }}$ and $\mathcal{D}_{\text {aux }}$ and choosing carefully $\bar{s}_{j}$ and $\bar{x}_{j}^{w}$, for every $j \in K$, we could take a full step in direction $(\Delta x, \Delta y, \Delta s)$ recovering primal and dual feasibilities. Now, we will analyse the consequences that such warmstarting approach has in the complementarity products. 


\subsection{Centrality}

The main motivation why we have chosen to minimize a variation of the weighted-least squares approach in our linear optimization problems is to avoid having large variations $(\Delta x, \Delta s)$ on small components. We would like to keep the terms $\left|\Delta x_{j}\right| /\left|x_{j}^{0}\right|$ and $\left|\Delta s_{j}\right| /\left|s_{j}^{0}\right|$ for every $j \in N$ bounded by some constant so that we could have some control on the centrality of the warmstarting point and therefore, a control on the new duality gap.

To analyse what the effect of our warmstarting strategy is considering centrality of the new iterate, let us first determine the depth of the cut for which our analysis holds. As stated before, the depth of cut $j$ is defined by $-\left(\bar{c}_{j}-\bar{A}_{j}^{T} y^{0}\right)$ for every $j \in K$. It is not surprising that if the depth of the cuts is large, we will need to backtrack to an earlier iterate. This is considered in our analysis by the notion of $\mu_{0}$ which is a reference barrier term that measures the depth of the cuts. When cuts are deep, $\mu_{0}$ is large so we may need to backtrack far from optimality, but when the cuts get shallower, $\mu_{0}$ gets smaller and therefore, we could choose an iterate close to optimality. Now, let us state the relation between the depth of the cuts and $\mu_{0}$ using the following expression for every $j \in K$

$$
\underbrace{\frac{1}{\gamma} \mu_{0} \frac{\sqrt{k} \mathcal{U}_{v} \sigma_{\max }}{\left(1-\delta_{l}\right) \sigma_{\min }(A)} \max \left\{\mu_{0}, \frac{1}{\mu_{0}}\right\}}_{:=\mathcal{U}_{c}} \geq \bar{c}_{j}-\bar{A}_{j}^{T} y^{0} \geq-\gamma \mu_{0} \frac{\bar{A}_{\min }}{\sqrt{m} \mathcal{U}_{v} \sigma_{\max }} .
$$

Note that we have some control with regards to the size of the cut. This means that we could retreat further back in the list of saved iterates to find a suitable large enough $\mu_{0}$ and the corresponding $\left(x^{0}, y^{0}, s^{0}\right)$ solution for which this condition is satisfied. Observe that increasing $\mu_{0}$ will expand both sides of inequality (29) increasing the chances to satisfy this condition. Also, observe that the left hand side inequality provides us with a very loose upper bound when by retreating back in the list of stored iterates, some of the reduced costs become positive. This bound becomes useful when defining the upper bound of the complementarity products obtained by our warmstarting strategy. Finally, it is fair to say that in some iterations (29) may not be satisfied by any of the stored iterates and therefore, we use coldstart instead.

Now, using definition (29) and the definition of $\bar{s}_{j}$ in (12), from (14) we deduce for every $j \in K$ that

$$
\begin{aligned}
d_{j} & =-\left(\bar{c}_{j}-\bar{A}_{j}^{T} y^{0}\right)+\bar{s}_{j}, \\
& \leq 2 \gamma \mu_{0} \frac{\bar{A}_{\min }}{\sqrt{m} \mathcal{U}_{v} \sigma_{\max }} .
\end{aligned}
$$

Hence,

$$
d_{\max } \leq 2 \gamma \mu_{0} \frac{\bar{A}_{\min }}{\sqrt{m} \mathcal{U}_{v} \sigma_{\max }}
$$


Using (30) in (18) and (19) we obtain

$$
\Delta s_{j} \leq 2 \gamma \mu_{0} \frac{1}{\mathcal{U}_{v}}, \forall j \in N
$$

and

$$
\bar{s}_{j}+\Delta \bar{s}_{j} \leq 2 \gamma \mu_{0} \frac{1}{\mathcal{U}_{v}}+\mathcal{U}_{c}, \quad \forall j \in K,
$$

respectively, where $\mathcal{U}_{c}$ denotes the left hand side term of inequality (29).

Now, let us analyse the consequences of our choices of $\bar{s}^{w}$ and $\bar{x}^{w}$ and their impact on the complementarity products of the warmstarting iterate. To do so, first let us define $\delta_{l}$ as

$$
\delta_{l}:=\min \left\{\frac{\mathcal{C}}{\mu_{0}+\mathcal{C}}, \frac{\mathcal{C}}{\frac{1}{\mu_{0}}+\mathcal{C}}\right\}
$$

where

$$
\mathcal{C}:=\frac{\gamma \sigma_{\min }(A) \bar{A}_{\min }}{\sqrt{k} \sqrt{m} \mathcal{U}_{v}^{2} \sigma_{\max }^{2}} .
$$

From (33) it is clear that $0<\delta_{l}<1$. Additionally, let us define $\delta_{u}$ as

$$
\delta_{u}:=6
$$

These values represent the coefficients used to expand the neighbourhood from below and above, respectively. As mentioned earlier, we expect the new warmstarting point to be inside a modified neighbourhood. Note that the parameter $\delta_{l}$ contains valuable information regarding the old problem and the new columns appended to it, through matrices $A$ and $\bar{A}$. Also, note that $\delta_{u}$ is problem independent. As we will discuss later, this is one of the key results of the analysis since it guarantees that regardless the size of the cut, and if some conditions hold, the new duality gap will be bounded by the old duality gap and a constant.

The following theorem states conditions and analyses complementarity products when our specialized warmstarting strategy is used.

Theorem 1 Let assumptions (A.1)-(A.4) hold and let $\left(x^{0}, y^{0}, s^{0}\right) \in \mathcal{N}_{s}(\gamma)$ with $\gamma \in(0,1)$. Additionally, let $\delta_{l}$ and $\delta_{u}$ be defined by (33) and $(35)$, respectively. Also, let us set

$$
\bar{x}_{j}^{w}=\gamma\left(1-\delta_{l}\right) \frac{\sigma_{\min }(A)}{\sqrt{k} \mathcal{U}_{v} \sigma_{\max }} \min \left\{\mu_{0}, \frac{1}{\mu_{0}}\right\}, \quad \forall j \in K,
$$

and

$$
\bar{s}_{j}=\max \left\{\gamma \mu_{0} \frac{\bar{A}_{\min }}{\sqrt{m} \mathcal{U}_{v} \sigma_{\max }}, \bar{c}_{j}-\bar{A}_{j}^{T} y^{0}\right\}, \quad \forall j \in K
$$


Having $\bar{c}_{j}-\bar{A}_{j}^{T} y^{0}$ satisfying (29), for every $j \in K$, and choosing $\Delta x$ from (21) and $(\Delta y, \Delta s)$ from (13) where $\Delta y=-\Delta \bar{y}$, we have that

$$
\left(x^{w}, \bar{x}^{w}, y^{w}, s^{w}, \bar{s}^{w}\right) \in \overline{\mathcal{N}}_{s}(\gamma),
$$

where

$\overline{\mathcal{N}}_{s}(\gamma)=\left\{\left(x^{w}, \bar{x}^{w}, y^{w}, s^{w}, \bar{s}^{w}\right) \in \overline{\mathcal{F}}: \delta_{l} \gamma \mu_{0} \leq x_{j}^{w} s_{j}^{w} \leq \delta_{u} \frac{1}{\gamma} \mu_{0}, \forall j \in N ; \delta_{l} \gamma \mu_{0} \leq \bar{x}_{k}^{w} \bar{s}_{k}^{w} \leq \delta_{u} \frac{1}{\gamma} \mu_{0}, \forall k \in K\right\}$,

and

$\overline{\mathcal{F}}=\left\{\left(x^{w}, \bar{x}^{w}, y^{w}, s^{w}, \bar{s}^{w}\right): A x^{w}+\bar{A} \bar{x}^{w}=b ; A^{T} y^{w}+s^{w}=c ; \bar{A}^{T} y^{w}+\bar{s}^{w}=\bar{c} ;\left(x^{w}, s^{w}\right)>0 ;\left(\bar{x}^{w}, \bar{s}^{w}\right)>0\right\}$.

Proof It is not difficult to show that by choosing $\delta_{l}$ from (33) and $\delta_{u}$ from (35), and using our previous choices for $\bar{x}_{j}^{w}$ (see (27)), and $\bar{s}_{j}^{w}=\bar{s}_{j}+\Delta \bar{s}_{j}$ (see (12) and (32)), the complementarity products for the new components, namely $\bar{x}_{j}^{w} \bar{s}_{j}^{w}$ for every $j \in K$, are inside the modified neighbourhood, $\overline{\mathcal{N}}_{s}(\gamma)$. Let us prove first that the upper bound holds. For every $j \in K$, we have that

$$
\begin{aligned}
\bar{x}_{j}^{w} \bar{s}_{j}^{w} & =\bar{x}_{j}^{w}\left(\bar{s}_{j}+\Delta \bar{s}_{j}\right), \\
& \leq \gamma\left(1-\delta_{l}\right) \frac{\sigma_{\min }(A)}{\sqrt{k} \mathcal{U}_{v} \sigma_{\max }} \min \left\{\mu_{0}, \frac{1}{\mu_{0}}\right\}\left(2 \gamma \mu_{0} \frac{1}{\mathcal{U}_{v}}+\mathcal{U}_{c}\right) .
\end{aligned}
$$

Since $0<\gamma<1,0<\delta_{l}<1, \mathcal{U}_{v}^{2}>1, k \geq 1, \min \left\{\mu_{0}, 1 / \mu_{0}\right\} \leq 1$, and the definition of $\mathcal{U}_{c}$, it is clear that

$$
\begin{aligned}
\bar{x}_{j}^{w} \bar{s}_{j}^{w} & \leq 2 \gamma \mu_{0} \frac{1}{\mathcal{U}_{v}} \gamma\left(1-\delta_{l}\right) \frac{\sigma_{\min }(A)}{\sqrt{k} \mathcal{U}_{v} \sigma_{\max }} \min \left\{\mu_{0}, \frac{1}{\mu_{0}}\right\}+\mu_{0}, \\
& <\delta_{u} \mu_{0}, \quad \forall j \in K,
\end{aligned}
$$

holds. If we now consider the lower bound for the complementarity products of the new components, we have that for every $j \in K$

$$
\begin{aligned}
\bar{x}_{j}^{w} \bar{s}_{j}^{w} & =\bar{x}_{j}^{w}\left(\bar{s}_{j}+\Delta \bar{s}_{j}\right), \\
& \geq \gamma\left(1-\delta_{l}\right) \frac{\sigma_{\min }(A)}{\sqrt{k} \mathcal{U}_{v} \sigma_{\max }} \min \left\{\mu_{0}, \frac{1}{\mu_{0}}\right\}\left(\gamma \mu_{0} \frac{\bar{A}_{\min }}{\sqrt{m} \mathcal{U}_{v} \sigma_{\max }}\right) .
\end{aligned}
$$

Using (34), we get

$$
\bar{x}_{j}^{w} \bar{s}_{j}^{w} \geq \gamma \mathcal{C}\left(1-\delta_{l}\right) \mu_{0} \min \left\{\mu_{0}, \frac{1}{\mu_{0}}\right\}, \quad \forall j \in K .
$$

Then, by choosing $\delta_{l}$ from (33), we have

$$
\bar{x}_{j}^{w} \bar{s}_{j}^{w} \geq \delta_{l} \gamma \mu_{0}, \quad \forall j \in K,
$$

Hence, and considering $\gamma \in(0,1),(36)$ and (37) the following result holds

$$
\delta_{l} \gamma \mu_{0} \leq \bar{x}_{j}^{w} \bar{s}_{j}^{w} \leq \delta_{u} \frac{1}{\gamma} \mu_{0}, \quad \forall j \in K,
$$


which completes the first part of the prove.

Now, we need to prove that the complementarity products of the old components, namely $x_{j}^{w} s_{j}^{w}$ for every $j \in N$, are inside the modified neighbourhood, $\overline{\mathcal{N}}_{s}(\gamma)$. Firstly, let us prove that the upper bound holds for every $j \in N$. By definition and conditions (8) and (28), we have that for every $j \in N$

$$
\begin{aligned}
x_{j}^{w} s_{j}^{w} & =x_{j}^{0} s_{j}^{0}\left(1+\frac{\Delta x_{j}}{x_{j}^{0}}\right)\left(1+\frac{\Delta s_{j}}{s_{j}^{0}}\right), \\
& \leq x_{j}^{0} s_{j}^{0}(2)\left(1+\Delta s_{j} \frac{\mathcal{U}_{v}}{\gamma \mu_{0}}\right) .
\end{aligned}
$$

Hence, and by using (31), we get

$$
x_{j}^{w} s_{j}^{w} \leq \delta_{u} \frac{1}{\gamma} \mu_{0}, \quad \forall j \in N .
$$

To prove that the lower bound holds we recall constraints $(21 \mathrm{c})$ which guarantee

$$
\Delta x_{j} \geq x_{j}^{0}\left(\delta_{l}-1\right), \quad \forall j \in N .
$$

Also, from (11), $\Delta s_{j} \geq 0$. Hence for every $j \in N$

$$
\begin{aligned}
x_{j}^{w} s_{j}^{w} & =x_{j}^{0} s_{j}^{0}\left(1+\frac{\Delta x_{j}}{x_{j}^{0}}\right)\left(1+\frac{\Delta s_{j}}{s_{j}^{0}}\right), \\
& \geq x_{j}^{0} s_{j}^{0}\left(1+\frac{\Delta x_{j}}{x_{j}^{0}}\right) .
\end{aligned}
$$

From (39) and since $x_{j}^{0}>0$, we know that

$$
1+\frac{\Delta x_{j}}{x_{j}^{0}} \geq \delta_{l}, \quad \forall j \in N .
$$

Replacing this in (40) gives

$$
x_{j}^{w} s_{j}^{w} \geq \delta_{l} x_{j}^{0} s_{j}^{0}, \quad \forall j \in N,
$$

which completes the proof.

\subsection{Algorithm}

Now we are in position to describe the algorithm proposed to find a warmstarting point after cuts of type $\bar{c}_{j}-\bar{A}_{j}{ }^{T} y<0$ are appended to the RMP. Note that this algorithm is embedded inside a major algorithm which is the primal-dual column generation method (PDCGM for short) [15]. Let us define $T=\{1,2, \ldots, h\}$ as the set of indexes of iterates which are strictly feasible and well-centred in the initial problem. Observe that $T, h$ and their corresponding list of stored solutions vary at each outer iteration of PDCGM. The list is created in ascending order so the last stored iterate and the closest-to-optimality 
$\overline{\text { Algorithm } 1 \text { Specialized warmstarting strategy for the primal-dual column }}$ generation method

Step 1 : Input $A, b, c, \bar{c}, \bar{A}, \mathcal{U}_{v}, n, k, m, \gamma \in(0,1)$, list of $\left(x^{t}, y^{t}, s^{t}\right) \in \mathcal{N}_{s}(\gamma)$ and $\mu_{t}=$ $\left(x^{t}\right)^{T} s^{t} / n$, where $t \in T$.

Step 2 : If no column is returned by the oracle, set $\left(x^{w}, y^{w}, s^{w}\right)=\left(x^{h}, y^{h}, s^{h}\right)$, and then go to Step 9. Otherwise, go to Step 3.

Step 3 : Calculate the smallest $\mu$ from the list of stored iterates such that

$$
\frac{1}{\gamma} \mu_{0} \frac{1}{\left(1-\delta_{l}\right)} \frac{\sqrt{k} \mathcal{U}_{v} \sigma_{\max }}{\sigma_{\min }(A)} \max \left\{\mu_{0}, \frac{1}{\mu_{0}}\right\} \geq \bar{c}_{j}-{\overline{A_{j}}}^{T} y^{0} \geq-\gamma \mu_{0} \frac{\bar{A}_{\min }}{\sqrt{m} \mathcal{U}_{v} \sigma_{\max }}
$$

is satisfied. If there exists such $\mu$, define $\mu=\mu_{0}$, denote its associated solution as Step 4 : Define $\left(x^{0}, y^{0}, s^{0}\right)$, and go to Step 4 . If not, use coldstart and go to Step 9.

$$
\delta_{l}:=\min \left\{\frac{\mathcal{C}}{\mu_{0}+\mathcal{C}}, \frac{\mathcal{C}}{\frac{1}{\mu_{0}}+\mathcal{C}}\right\}, \text { where } \mathcal{C}:=\frac{\gamma \bar{A}_{\min } \sigma_{\min }(A)}{\sqrt{k} \sqrt{m} \mathcal{U}_{v}^{2} \sigma_{\max }^{2}}
$$

Step 5 : Set for every $j \in K$

$$
\begin{aligned}
& \bar{s}_{j}=\max \left\{\gamma \mu_{0} \frac{\bar{A}_{\text {min }}}{\sqrt{m} \mathcal{U}_{v} \sigma_{\max }}, \bar{c}_{j}-\bar{A}_{j}^{T} y^{0}\right\}, \\
& \bar{x}_{j}^{w}=\gamma\left(1-\delta_{l}\right) \frac{\sigma_{\min }(A)}{\sqrt{k} \mathcal{U}_{v} \sigma_{\max }} \min \left\{\mu_{0}, \frac{1}{\mu_{0}}\right\},
\end{aligned}
$$

and define $\mathcal{B}_{0}$ and $\mathcal{N}_{0}$.

Step 6 : Solve $\mathcal{D}_{\text {aux }}$. Output: $(\Delta y, \Delta s, \Delta \bar{s})$.

Step 7 : Solve $\mathcal{P}_{\text {aux }}$. Output: $\Delta x$.

Step $8:$ Define $\left(x^{w}, \bar{x}^{w}, y^{w}, s^{w}, \bar{s}^{w}\right)=\left(x^{0}+\Delta x, \bar{x}^{w}, y^{0}+\Delta y, s^{0}+\Delta s, \bar{s}+\Delta \bar{s}\right)$.

Step 9 : Continue with the usual primal-dual interior point method by solving the Newton system of equations in the old/new dimensions.

solution is denoted by $\left(x^{h}, y^{h}, s^{h}\right)$. Algorithm 1 summarizes the principal steps of our specialized warmstarting strategy.

Observe that a new list of stored iterates is used every time we initialize Algorithm 1. Note that Step 2 accounts for the case in which no columns with negative reduced costs are obtained from the oracle. This is a particular feature of the PDCGM. There is no point of using a warmstarting strategy since the current iterate is already well-centred and strictly feasible and therefore starting from it is the best we could do. Also, it is important to note that every cut $\bar{c}_{j}-\bar{A}_{j}{ }^{T} y^{h}<0$ is generated using $\left(x^{h}, y^{h}, s^{h}\right)$. Hence, for any iterate $\left(x^{t}, y^{t}, s^{t}\right)$, where $t \in T$, some of the new columns (cuts) may actually become dual feasible, or at least less infeasible (shallower cuts). The following theorem summarizes the main discussion and results of this section.

Theorem 2 Using Algorithm 1 and given that a suitable $\left(x^{0}, y^{0}, s^{0}\right)$ is available from the list of iterates, the solution $\left(x^{w}, \bar{x}^{w}, y^{w}, s^{w}, \bar{s}^{w}\right) \in \overline{\mathcal{N}_{s}}(\gamma)$ and the new duality gap is bounded by

$$
\left(x^{w}\right)^{T} s^{w}+\left(\bar{x}^{w}\right)^{T} \bar{s}^{w} \leq 6(n+k) \frac{1}{\gamma} \mu_{0} .
$$


Proof The proof follows from Lemmas 2 and 4 and Theorem 1.

In summary, our strategy aims to restore primal and dual feasibilities separately by means of auxiliary linear optimization problems. We can ensure that primal and dual feasibilities are fully recovered if a suitable $\mu$ can be found. Note that Theorem 2 provides a guarantee that the new duality gap is bounded by the old duality gap multiplied with a constant which depends on the number of columns added to the old problem. Our analysis takes into account special classes of problems arising in combinatorial optimization with non-negative technological coefficients.

\section{Numerical experiments}

We have implemented the proposed warmstarting strategy inside the primaldual column generation method described in [15]. This column generation implementation has a built-in procedure which allows to store an advanced iterate in order to use it for future warmstart. We have taken advantage of this to implement our strategy. We have tested three different strategies to solve the relaxation of the one-dimensional cutting stock problem (CSP) and the vehicle routing problem with time windows (VRPTW) after applying the Dantzig-Wolfe reformulation [5]. CSP problem consists in the minimization of the number of rolls of fixed width that have to be cut in order to satisfy the demands of different pieces with different widths [10]. VRPTW deals with the problem of minimizing the distance travelled for a fleet of vehicles while delivering products to a set of customers distributed in a given area. Additionally, capacity of each vehicle and a time window when a vehicle should deliver the product are considered [22]. For a mathematical description of these problems and their corresponding decomposition, the reader is referred to [2].

The strategies considered to find a suitable candidate from which to start solving each RMP are:

- Coldstart (CS). It refers to solving a given RMP without considering any information of the previously solved RMP and therefore, every RMP is solved from scratch. We rely on the presolving and heuristic procedures included in HOPDM [13].

- Partial feasibility restoration (PFR). This strategy was introduced in [14] and has been successfully applied to speed up the column generation procedure in $[15,18]$. As described in Section 4, this strategy ensures that dual feasibility is fully restored in the new components if cuts are not too deep. However, it does not ensure the same in the old components. Additionally, it sets the new components in the primal space to a small value so primal feasibility can be easily restored.

- One step primal-dual restoration (1SPDR). This strategy has been described in this paper and aims to recover primal and dual feasibilities in one step by solving two auxiliary problems. Also, theoretical guarantees are provided so the warmstarting iterate stays close to the new central path. 
The subproblems obtained after applying the reformulation to CSP and VRPTW are solved using the same source code, i.e., knapsack solver [24] (CSP) and our own implementation of the bounded bidirectional dynamic programming algorithm proposed in [32], with state-space relaxation and identification of unreachable nodes [7] (VRPTW). For the three aforementioned strategies (CS, PFR and 1SPDR), the RMPs are initialized with the same columns. However, after the first iteration every RMP may be different and therefore, we may expect that some of the strategies will require fewer outer iterations than others. To run the tests we have used a laptop with a $2.30 \mathrm{Ghz}$ Intel Core i5 processor, 6 GB RAM and a Linux operating system. The implementations have been developed in $\mathrm{C}$ (solving the two auxiliary problems) and FORTRAN (HOPDM native language). For each of the strategies, we stop the column generation procedure when the relative duality gap becomes smaller than the default accuracy $\delta=10^{-6}$.

In the results presented in [15], it was observed that the time spent solving the RMP was considerable when compared with the time spent in the oracle when solving CSP. On the other hand, the dominant task when solving VRPTW was the oracle and the RMP time only accounted for a small portion of the total time. By presenting the results for these two applications, we could get a better understanding of the situations where we can expect a warmstarting strategy to perform better than coldstart and how much the gain can be.

Before continuing, it is important to give some remarks about our implementation. Note that for advanced column generation iterations, the coefficients of the objective function, in the primal and dual auxiliary problems $\left(1 / s_{j}^{0}\right.$ in $(11)$ and $1 / x_{j}^{0}$ in $\left.(21)\right)$, can lead to very badly-scaled problems (some very large coefficients and others very small). Therefore, we have decided to scale these coefficients and restrict them to a narrower range. In other words, if a given coefficient is greater/smaller than a predefined threshold, we have used this threshold as the coefficient for this specific variable. Note that by doing this, we do not affect the primal and dual feasible sets, only the scaling of that particular auxiliary problem and therefore, the strategy still recovers primal and dual feasibility in one step. Additionally, since PDCGM is based on HOPDM [13], an infeasible primal-dual interior point method is used to solve every RMP which keeps the iterates inside a neighbourhood of the central path by making use of multiple centrality corrector steps [3]. We have considered this in our developments in the following way. When the column generation process approaches optimality and the depth of the cuts is small, that is when $\mu$ and $\left(\bar{c}_{j}-\bar{A}_{j}^{T} y_{j}^{0}\right)$ are small, instead of solving the primal and dual auxiliary problems, we set the values of new components to $\bar{x}_{j}^{w}=\sqrt{\mu / \gamma}$ and $\bar{s}_{j}^{w}=\sqrt{\gamma \mu}$ for every $j \in K$. We keep the old iterates unchanged. This choice is justified since the cuts at this stage are likely to be shallow and therefore, by choosing the new components in this way, we only generate small infeasibilities which HOPDM (and any infeasible primal-dual interior point method) can easily handle. Additionally, with these choices, we ensure that the complementarity 
products for the new iterates are inside the neighbourhood described in (7). Moreover, and after some testing, the directions, and therefore, the correction steps, obtained by solving the auxiliary problems in an advanced stage of the column generation process, are very short and do not change significantly the stored iterate. By performing this small change in the algorithm, we aim to reduce the CPU time of the method by avoiding unnecessary calls to the auxiliary problems and taking advantage of the infeasible primal-dual interior point method. Finally, to solve the primal and dual auxiliary problems we rely on the solver HOPDM with tolerance set to $10^{-4}$.

\subsection{The cutting stock problem (CSP)}

To analyse the performance of these three strategies for solving CSP, we have selected 262 instances from the literature in the one-dimensional CSP (http://www.math.tu-dresden.de/ capad/). The size of the instances vary between 15 and 585 items. The column generation procedure is initialized with columns generated by homogeneous cutting patterns, which corresponds to selecting only one piece per pattern, as many times as possible without violating the width of the rolls.

In Table 1, we summarize our findings. In the first column we denote by $k$ the number of columns added at each iteration of the column generation procedure. Note that by adding more columns at every iteration we are targeting to reduce the number of calls to the oracle. However, in terms of warmstarting this may have an important impact since the similarities between two consecutive RMPs are likely to be lost. We have grouped the instances in two classes, denoted by Easy and Hard. The former contains 97 instances while the latter 165 instances. In row All, we have included the average results when all the instances are considered for that particular value of $k$. For each strategy we show the average number of inner iterations (inner) and the average CPU time required to solve the RMPs (rmp). Inner iterations refer to the total number of iterations required to solve the RMPs while the RMP time considers the time required to solve the RMPs and the time of any warmstating procedure. For instance, for 1SPDR, it is the overall time of solving the primal and dual auxiliary problems and the time of solving each RMP. Moreover, we also include the total time required for the PDCGM to converge to an optimal solution (tot).

Note that the best performance in terms of CPU time for each of the strategies and each group of instances is obtained when 10 columns are added at each iteration. From our results it seems that by adding 10 columns, the number of column generation iterations is reduced considerably when compared to a single-column approach and at the same time, each strategy (re)initializes and solves the RMPs efficiently. Note that considering all instances, the best performance is achieved by 1SPDR when adding 10 columns. It is also fair to say that the performance of PFR is comparable to the one proposed in this study when this number of columns is added per iteration. Both warmstarting 
Table 1 Average results on 262 instances of CSP for PFR, CS and 1SPDR strategies adding $k$ columns at a time: RMP iterations and times (RMP and total).

\begin{tabular}{|c|c|c|c|c|c|c|c|c|c|c|}
\hline \multirow[b]{3}{*}{$\mathrm{k}$} & \multirow[b]{3}{*}{ class } & \multicolumn{3}{|c|}{ PFR } & \multicolumn{3}{|c|}{ CS } & \multicolumn{3}{|c|}{ 1SPDR } \\
\hline & & \multirow[b]{2}{*}{ inner } & \multicolumn{2}{|c|}{ times $(\mathrm{s})$} & \multirow[b]{2}{*}{ inner } & \multicolumn{2}{|c|}{ times $(\mathrm{s})$} & \multirow[b]{2}{*}{ inner } & \multicolumn{2}{|c|}{ times $(\mathrm{s})$} \\
\hline & & & $\mathrm{rmp}$ & tot & & $\mathrm{rmp}$ & tot & & $\mathrm{rmp}$ & tot \\
\hline \multirow{3}{*}{1} & Easy & 1303.9 & 3.9 & 4.9 & 3504.2 & 7.8 & 8.7 & 1762.5 & 4.1 & 4.8 \\
\hline & Hard & 3105.6 & 15.0 & 38.2 & 6184.4 & 37.0 & 66.4 & 3288.0 & 15.2 & 36.8 \\
\hline & All & 2438.5 & 10.9 & 25.9 & 5192.1 & 26.2 & 45.0 & 2723.2 & 11.1 & 25.0 \\
\hline \multirow{3}{*}{10} & Easy & 427.3 & 1.6 & 2.1 & 1082.4 & 3.4 & 3.9 & 600.4 & 1.9 & 2.4 \\
\hline & Hard & 1002.7 & 6.1 & 15.5 & 2080.9 & 13.7 & 23.9 & 1118.7 & 6.7 & 14.6 \\
\hline & All & 789.7 & 4.4 & 10.6 & 1711.2 & 9.9 & 16.5 & 926.8 & 5.0 & 10.1 \\
\hline \multirow{3}{*}{50} & Easy & 308.5 & 2.1 & 3.9 & 732.3 & 4.1 & 5.6 & 437.9 & 2.3 & 3.6 \\
\hline & Hard & 706.0 & 8.5 & 24.4 & 1540.5 & 18.2 & 32.6 & 1064.4 & 11.9 & 23.2 \\
\hline & All & 558.8 & 6.2 & 16.8 & 1241.3 & 13.0 & 22.6 & 832.5 & 8.3 & 15.9 \\
\hline \multirow{3}{*}{100} & Easy & 278.6 & 1.8 & 4.6 & 667.5 & 4.8 & 7.9 & 397.4 & 2.9 & 5.7 \\
\hline & Hard & 665.3 & 9.0 & 33.4 & 1454.8 & 26.1 & 53.6 & 1059.0 & 20.2 & 44.1 \\
\hline & All & 522.1 & 6.3 & 22.8 & 1163.3 & 18.2 & 36.7 & 814.0 & 13.8 & 29.9 \\
\hline
\end{tabular}

Table 2 Average results on 262 instances of CSP for PFR, CS and 1SPDR adding $k$ columns at a time: column generation calls, inner iterations and time per RMP.

\begin{tabular}{crrrrrrrrr}
\hline \multicolumn{3}{c}{ PFR } & \multicolumn{3}{c}{ CS } & \multicolumn{3}{c}{ 1SPDR } \\
\hline $\mathrm{k}$ & $\mathrm{rmp}$ & inner/rmp & time/rmp & $\mathrm{rmp}$ & inner/rmp & time/rmp & $\mathrm{rmp}$ & inner/rmp time/rmp \\
\hline 1 & 440.4 & 5.5 & 0.02 & 487.5 & 10.7 & 0.05 & 423.2 & 6.4 & 0.03 \\
10 & 120.0 & 6.6 & 0.04 & 146.2 & 11.7 & 0.07 & 111.9 & 8.3 & 0.04 \\
50 & 73.8 & 7.6 & 0.08 & 91.9 & 13.5 & 0.14 & 67.5 & 12.3 & 0.12 \\
100 & 62.8 & 8.3 & 0.10 & 78.6 & 14.8 & 0.23 & 59.9 & 13.6 & 0.23 \\
\hline
\end{tabular}

strategies outperform a coldstart approach in both number of inner iterations and CPU times.

Let us now compare CS and 1SPDR. Since 1SPDR successfully restores primal and dual feasibility in almost all the column generation iterations and keeps the complementarity products of the warmstarting iterate inside a slightly modified neighbourhood, the number of inner iterations to solve the new problem is reduced when compared to CS. The reductions in inner iterations using 1SPDR vary in average between $30 \%(k=100)$ and $48 \%(k=1)$ with respect to the inner iterations required by CS. To calculate these reductions we have used $\left(1-\frac{\text { inner }_{1 S P D R}}{\text { inner }_{C S}}\right) \times 100 \%$. The savings in time are due to the reduction of inner iterations and also the efficiency of calculating the new warmstarted iterate. The gains in total CPU time by using 1SPDR strategy range between $19 \%$ to $45 \%$ ( $k=100$ and $k=1$, respectively). These gains were determined considering $\left(1-\frac{\text { total }_{1 S P D R}}{\text { total }_{C S}}\right) \times 100 \%$.

The differences between 1SPDR and PFR are due to the nature of each of these strategies and the environment for which they were developed. It is necessary to remark that $1 \mathrm{SPDR}$ aims to recover primal and dual feasibility while keeping the warmstarted iterate inside a slightly modified neighbour- 
hood, but PFR aims to recover only feasibility in the new dual components and does not ensure the same for the old components. Additionally, the latter was designed to take full advantage of an infeasible primal-dual interior point method while the former has being developed considering a feasible primaldual interior point method and only takes advantage of the infeasibility nature of the solver at the end of the column generation process, namely when the primal-dual column generation method is close to termination. In terms of inner iterations, PFR outperforms 1SPDR. This may be due to the nature of PFR, which delivers close to feasible solutions but at the same time, the iterates are far enough from the boundaries. Then, only few centering steps are needed to recover feasibility and return to the neighbourhood of the new central path. On the other hand, 1SPDR ensures primal and dual feasibility restorations. However, and as a consequence of such restorations, it slightly increases the neighbourhood and therefore, at some iterations the warmstarting iterate may be too close to the boundaries. This originates a sometimes excessive number of centering steps in order to return to the neighbourhood of the new central path increasing the number of inner iterations. It is important to point out that this belief is just empirical since no theoretical support is given for PFR with respect to complementarity after the restoration is performed. In terms of CPU time, it is not clear which method performs better. However, the best performance in terms of total CPU time is obtained when using 1 SPDR when 10 columns are added.

Additionally, in Table 2 we present more results of these experiments considering all the instances. In this table, we include the average number of column generation iterations ( $\mathrm{rmp}$ ), the average number of inner iterations per RMP (inner/rmp) and the average CPU time required to solve every RMP (time/rmp) which is expressed in seconds per RMP.

It is clear from Table 2 that by adding more columns to the RMP at each iteration $(k)$, the number of outer iterations is reduced for all the strategies. A similar result was obtained in [15] by using the PFR strategy in the PDCGM and for other applications such as the vehicle routing problem with time windows and the capacitated lot-sizing problem with setup times. An important benefit of using 1SPDR over PFR and CS with respect to the number of outer iterations can be observed from Table 2 for CSP. We believe that for this application taking care of centrality aspects, as with 1SPDR, stabilizes even more the PDCGM and therefore, less calls to the oracle are needed. In other words, the duals sent to the oracle using 1SPDR are better centred than the ones obtained by the other two strategies reducing the column generation iterations. As a consequence of this, the time spent in the oracle is reduced. This is the main reason why $1 \mathrm{SPDR}$ is comparable with the PR in terms of total CPU time. Although adding more columns at each iteration reduces the number of calls to the oracle (which is the most time consuming task for this application), it makes the task of warmstarting more difficult since the new RMP is likely to be very different from the old RMP. This can be seen in the average time required to solve the RMPs. The speed-ups obtained by using any 
of the warmstarting strategies presented here are considerable when compared against CS no matter the number of columns added per iteration.

\subsection{The vehicle routing problem with time windows (VRPTW)}

From VRPTW literature we have selected 87 instances (http://www2.imm. $\mathrm{dtu} . \mathrm{dk} / \sim \mathrm{jla} /$ solomon.html) originally proposed in [34]. We have used the number of customers to classify the instances in three groups. Class $S$ corresponds to instances with 25 customers, while class $M$ with 50 customers and class $L$ contains instances with 100 customers. The column generation procedure is initialized with $n$ single-customer routes (where $n$ denotes the number of customers), which corresponds to assigning one vehicle per customer.

Table 3 Average results on 87 instances of VRPTW for PFR, CS and 1SPDR adding $k$ columns at a time: RMP iterations and times (RMP and total).

\begin{tabular}{|c|c|c|c|c|c|c|c|c|c|c|}
\hline \multirow[b]{3}{*}{$\mathrm{k}$} & \multirow[b]{3}{*}{ class } & \multicolumn{3}{|c|}{ PFR } & \multicolumn{3}{|c|}{$\mathrm{CS}$} & \multicolumn{3}{|c|}{ 1SPDR } \\
\hline & & \multirow[b]{2}{*}{ inner } & \multicolumn{2}{|c|}{ times $(\mathrm{s})$} & \multirow[b]{2}{*}{ inner } & \multicolumn{2}{|c|}{ times $(\mathrm{s})$} & \multirow[b]{2}{*}{ inner } & \multicolumn{2}{|c|}{ times $(\mathrm{s})$} \\
\hline & & & $\mathrm{rmp}$ & tot & & $\mathrm{rmp}$ & tot & & $\mathrm{rmp}$ & tot \\
\hline \multirow{4}{*}{1} & $\mathrm{~S}$ & 199.8 & 0.3 & 1.0 & 222.1 & 0.2 & 0.7 & 366.6 & 0.3 & 0.8 \\
\hline & M & 397.6 & 0.6 & 6.7 & 525.6 & 0.5 & 6.2 & 866.7 & 0.9 & 5.9 \\
\hline & $\mathrm{L}$ & 769.0 & 1.2 & 111.5 & 1109.9 & 1.4 & 116.3 & 2038.6 & 2.9 & 124.3 \\
\hline & All & 455.4 & 0.7 & 39.7 & 619.2 & 0.7 & 41.1 & 1090.6 & 1.4 & 43.7 \\
\hline \multirow{4}{*}{10} & $\mathrm{~S}$ & 132.1 & 0.2 & 0.4 & 115.5 & 0.1 & 0.4 & 175.5 & 0.2 & 0.4 \\
\hline & M & 242.4 & 0.4 & 2.6 & 206.6 & 0.3 & 2.5 & 336.2 & 0.4 & 2.5 \\
\hline & $\mathrm{L}$ & 470.7 & 0.8 & 36.7 & 414.9 & 0.8 & 38.3 & 772.7 & 1.5 & 38.4 \\
\hline & All & 281.7 & 0.5 & 13.2 & 245.7 & 0.4 & 13.7 & 428.1 & 0.7 & 13.8 \\
\hline \multirow{4}{*}{50} & $\mathrm{~S}$ & 115.1 & 0.2 & 0.3 & 115.5 & 0.2 & 0.4 & 152.2 & 0.2 & 0.4 \\
\hline & $\mathrm{M}$ & 208.9 & 0.4 & 1.8 & 177.0 & 0.4 & 2.1 & 242.7 & 0.5 & 1.9 \\
\hline & $\mathrm{L}$ & 405.2 & 1.2 & 23.2 & 361.5 & 1.6 & 27.9 & 579.2 & 2.1 & 24.5 \\
\hline & All & 243.1 & 0.6 & 8.4 & 218.0 & 0.8 & 10.1 & 324.7 & 0.9 & 8.9 \\
\hline \multirow{4}{*}{100} & $\mathrm{~S}$ & 113.6 & 0.2 & 0.3 & 118.8 & 0.2 & 0.4 & 146.5 & 0.3 & 0.4 \\
\hline & $\mathrm{M}$ & 184.7 & 0.4 & 1.6 & 166.8 & 0.5 & 1.9 & 244.0 & 0.7 & 1.9 \\
\hline & $\mathrm{L}$ & 348.6 & 1.5 & 18.3 & 291.7 & 1.7 & 18.4 & 458.5 & 2.5 & 19.8 \\
\hline & All & 215.7 & 0.7 & 6.8 & 192.4 & 0.8 & 6.9 & 283.0 & 1.2 & 7.3 \\
\hline \multirow{4}{*}{200} & $\mathrm{~S}$ & 120.0 & 0.3 & 0.4 & 120.0 & 0.3 & 0.5 & 144.2 & 0.4 & 0.5 \\
\hline & M & 186.6 & 0.6 & 1.7 & 178.5 & 0.8 & 2.0 & 249.4 & 1.0 & 2.0 \\
\hline & $\mathrm{L}$ & 359.3 & 2.3 & 17.2 & 296.3 & 2.6 & 19.0 & 445.3 & 3.5 & 18.3 \\
\hline & All & 222.0 & 1.1 & 6.4 & 198.3 & 1.3 & 7.2 & 279.6 & 1.6 & 7.0 \\
\hline \multirow{4}{*}{300} & $\mathrm{~S}$ & 119.8 & 0.3 & 0.5 & 128.2 & 0.4 & 0.6 & 147.0 & 0.4 & 0.6 \\
\hline & $\mathrm{M}$ & 197.2 & 0.9 & 2.0 & 182.0 & 1.1 & 2.2 & 254.9 & 1.2 & 2.2 \\
\hline & $\mathrm{L}$ & 379.1 & 3.0 & 16.6 & 285.8 & 3.2 & 18.1 & 469.1 & 4.6 & 18.3 \\
\hline & All & 232.0 & 1.4 & 6.3 & 198.6 & 1.6 & 7.0 & 290.3 & 2.1 & 7.0 \\
\hline
\end{tabular}

In Table 3 we present the results of our computational experiments for VRPTW. As in Section 5.1, the first two columns denote the number of 
Table 4 Average results on 87 instances of VRPTW for PFR, CS and 1SPDR adding $k$ columns at a time: column generation calls, inner iterations and time per RMP.

\begin{tabular}{crrrrrrrrr}
\hline \multicolumn{3}{c}{ PFR } & \multicolumn{3}{c}{ CS } \\
\hline $\mathrm{k}$ & $\mathrm{rmp}$ & inner/rmp & time/rmp & $\mathrm{rmp}$ & inner/rmp & time/rmp & $\mathrm{rmp}$ & inner/rmp & time/rmp \\
\hline 1 & 121.6 & 3.7 & 0.01 & 124.9 & 5.0 & 0.01 & 124.9 & 8.7 & 0.01 \\
10 & 44.4 & 6.3 & 0.01 & 45.7 & 5.4 & 0.01 & 45.2 & 9.5 & 0.02 \\
50 & 30.6 & 8.0 & 0.02 & 33.8 & 6.5 & 0.02 & 31.1 & 10.4 & 0.03 \\
100 & 25.6 & 8.4 & 0.03 & 28.8 & 6.7 & 0.03 & 26.1 & 10.8 & 0.04 \\
200 & 23.3 & 9.5 & 0.05 & 26.6 & 7.5 & 0.05 & 23.4 & 11.9 & 0.07 \\
300 & 22.2 & 10.4 & 0.06 & 25.4 & 7.8 & 0.06 & 22.0 & 13.2 & 0.09 \\
\hline
\end{tabular}

columns we aim to add per iteration to the RMP and the classification, respectively. We also present the average number of inner iterations (inner), the average RMP time in seconds ( $\mathrm{rmp}$ ) and the average total time (tot) per strategy and number of columns allowed to be added per iteration $(k)$.

As shown in [15], the most expensive task for this application with the PDCGM is solving the oracle (difference between the total and RMP times). When the number of columns added per iteration is small, the RMP time accounts as a small proportion of the total CPU time. Therefore, it might seem a bit odd to see that for some classes the total CPU time is slightly lower while the RMP time is slightly higher. This is only due to savings in the number of outer iterations and therefore, in oracle time. The importance of the RMP time with respect to the total CPU time increases with the number of columns allowed to be added per iteration. This can be explained since the RMP becomes computationally more expensive (the instance becomes larger and the warmstarting strategy procedure, if any, consumes time) while the total CPU time is reduced.

Similarly, in Table 4 we include more information to help with the interpretation of our results, namely, the average number of outer iterations ( $\mathrm{rmp}$ ), the average number of inner iterations per RMP (inner/rmp) and the average time required to solve every RMP (time/rmp) expressed in seconds per RMP.

At first it seems surprising that CS performs so well. One explanation is that the presolving techniques used by HOPDM are very effective for this class of problems. Note that when one column is added, the average number of inner iterations per RMP for CS is 5.0 (column inner/rmp in Table 4).

For this application and the instances chosen, the depth of the cut is usually very large when compared with the reference parameter $\mu$ and therefore, the method retreats back in the list of iterates moving far away from the region close to optimality and therefore few more steps are required to reach the desirable sub-optimal solution of the modified problem. However, the relative large number of inner iterations required for our strategy on average when compared to PFR and CS, does not have a significant impact on the efficiency of the method. If we compare the average time per iteration spent in solving the RMP, one can conclude that the RMP is solved very efficiently regardless the initial iterate (PFR, CS or $1 \mathrm{SPDR})$. 1SPDR requires slightly more time 
to solve the RMP since this approach has to solve two auxiliary problems. The difficulty in solving the RMP increases in all cases with the number of columns added per iteration. Note that for VRPTW and for the same number of columns added per iteration, the outer iterations remains almost constant, no matter if we use coldstart or any of the warmstarting strategies.

It is important to point out that the columns obtained for VRPTW are very different from the ones obtained for CSP. While for CSP, the columns represent cutting patterns which have non-zero integer entries and may be dense, VRPTW has columns containing only few non-zero integer entries representing routes. Due to the vehicle capacity and time window constraints one may expect that only few customers are served by each vehicle so sparse columns are common. On the other hand for CSP, the more challenging instances are the ones with large width allowing more dense columns.

The structure of VRPTW adversely affects 1SPDR. It has been observed that if only few components of a new column have non-zero entries, the set of possible directions which recovers primal and dual feasibilities may be very limited. Therefore, large variations in small components are expected and as a consequence of this, a bad performance of this strategy in practice.

Finally, from our computational experiments, one can conclude than in some situations, CS (understood as the initialization provided by HOPDM) may be competitive to an efficient warmstarting strategy. Nevertheless, we strongly believe that this is more an exception than the rule and therefore, warmstarting is indeed needed in this context. Note that for VRPTW all strategies studied here are efficient and solve each RMP in less than a tenth of a second.

\section{Conclusions}

In this paper we have presented a new strategy to warmstart a primal-dual interior point method in the context of column generation. The method deals with primal and dual feasibility restorations while keeping some control in the duality gap of the new warmstart. Proofs that the method recovers primal and dual feasibilities in one step after new columns are appended to the restricted master problem are provided. Also, conditions are given to guarantee that the new duality gap is bounded by a small multiple of the old one, see Theorem 2. Additionally, computational experiments for solving a reformulation of the cutting stock problem demonstrate the benefits of using such warmstarting strategy when compared to coldstart. In general, savings between $30 \sim 48 \%$ in the number of inner iterations and $19 \sim 45 \%$ in CPU time can be achieved on average for different column generation scenarios. For CSP, the advantages of the proposed method with respect to coldstart are consistent no matter the number of columns added to each restricted master problem at each iteration. Also, we have provided computational evidence that the proposed strategy and coldstart behave similarly in terms of CPU time for solving a reformulation of the vehicle routing problem with time windows. Both strategies seem 
to be very efficient due to the structure of the restricted master problems and the presolving embedded in HOPDM. Additionally, we have compared our strategy with the one presented in [14] which restores dual feasibility in the new components after new columns are added. Both warmstarting strategies are competitive in terms of CPU time for both applications. Further research directions are to analyse and develop a warmstarting strategy that considers primal and dual infeasibilities so we can take advantage of the infeasible primal-dual interior point solver HOPDM. Also, we plan to investigate more efficient ways of calculating directions in the primal and dual spaces.

Acknowledgements We would like to thank the referees for their useful comments and suggestions which allowed us to improve the quality and presentation of this paper. Pablo González-Brevis would like to thank the support given by Facultad de Ingeniería, Universidad del Desarrollo and Beca Presidente de la República (CONICYT) from Chile.

\section{References}

1. Benson, H.Y., Shanno, D.F.: An exact primal-dual penalty method approach to warmstarting interior-point methods for linear programming. Computational Optimization and Applications 38(3), 371-399 (2007)

2. Briant, O., Lemaréchal, C., Meurdesoif, P., Michel, S., Perrot, N., Vanderbeck, F.: Comparison of bundle and classical column generation. Mathematical Programming 113, 299-344 (2008)

3. Colombo, M., Gondzio, J.: Further development of multiple centrality correctors for interior point methods. Computational Optimization and Applications 41(3), 277-305 (2008)

4. Cornuejols, G., Fisher, M.L., Nemhauser, G.L.: Location of bank accounts to optimize float: An analytic study of exact and approximate algorithms. Management Science 23(8), 789-810 (1977)

5. Dantzig, G.B., Wolfe, P.: The decomposition algorithm for linear programs. Econometrica 29(4), 767-778 (1961)

6. Engau, A., Anjos, M.F., Vannelli, A.: On interior-point warmstarts for linear and combinatorial optimization. SIAM Journal on Optimization 20(4), 1828-1861 (2010)

7. Feillet, D., Dejax, P., Gendreau, M., Gueguen, C.: An exact algorithm for the elementary shortest path problem with resource constraints: application to some vehicle-routing problems. Networks 44, 216-229 (2004)

8. Fliege, J.: An efficient interior-point method for convex multicriteria optimization problems. Mathematics of Operations Research 31(4), 825-845 (2006)

9. Freund, R.M.: A potential-function reduction algorithm for solving a linear program directly from an infeasible "warm start". Mathematical Programming 52, 441-466 (1991)

10. Gilmore, P.C., Gomory, R.E.: A linear programming approach to the cutting-stock problem. Operations Research 9(6), 849-859 (1961)

11. Goffin, J.L., Haurie, A., Vial, J.P.: Decomposition and nondifferentiable optimization with the projective algorithm. Management Science 38(2), 284-302 (1992)

12. Goffin, J.L., Vial, J.P.: Multiple cuts in the analytic center cutting plane method. SIAM Journal on Optimization 11(1), 266-288 (2000)

13. Gondzio, J.: HOPDM (version 2.12) - a fast LP solver based on a primal-dual interior point method. European Journal of Operational Research 85, 221-225 (1995)

14. Gondzio, J.: Warm start of the primal-dual method applied in the cutting-plane scheme. Mathematical Programming 83, 125-143 (1998)

15. Gondzio, J., González-Brevis, P., Munari, P.: New developments in the primal-dual column generation technique. European Journal of Operational Research 224(1), 41$51(2013)$ 
16. Gondzio, J., Grothey, A.: Reoptimization with the primal-dual interior point method. SIAM Journal on Optimization 13(3), 842-864 (2003)

17. Gondzio, J., Grothey, A.: A new unblocking technique to warmstart interior point methods based on sensitivity analysis. SIAM Journal on Optimization 19(3), 1184-1210 (2008)

18. Gondzio, J., Sarkissian, R.: Column generation with a primal-dual method. Technical Report 96.6, Logilab (1996)

19. Held, M., Karp, R.M.: The traveling-salesman problem and minimum spanning trees. Operations Research 18(6), 1138-1162 (1970)

20. Held, M., Karp, R.M.: The traveling-salesman problem and minimum spanning trees: Part II. Mathematical Programming 1(1), 6-25 (1971)

21. John, E., Yildirim, E.A.: Implementation of warm-start strategies in interior-point methods for linear programming in fixed dimension. Computational Optimization and Applications 41, 151-183 (2008)

22. Kallehauge, B., Larsen, J., Madsen, O.B., Solomon, M.M.: Vehicle routing problem with time windows. In: G. Desaulniers, J. Desrosiers, M.M. Solomon (eds.) Column Generation, pp. 67-98. Springer US (2005)

23. Karmarkar, N.: A new polynomial-time algorithm for linear programming. Combinatorica 4, 373-395 (1984)

24. Leão, A.A.S.: Geração de colunas para problemas de corte em duas fases. Master's thesis, ICMC - University of Sao Paulo, Brazil (2009)

25. Lübbecke, M.E., Desrosiers, J.: Selected topics in column generation. Operations Research 53(6), 1007-1023 (2005)

26. Martinson, R.K., Tind, J.: An interior point method in Dantzig-Wolfe decomposition. Computers and Operations Research 26, 1195-1216 (1999)

27. Mitchell, J.E.: Karmarkar's algorithm and combinatorial optimization problems. Ph.D. thesis, School of Operations Research and Industrial Engineering, Cornell University (1988)

28. Mitchell, J.E., Borchers, B.: Solving real-world linear ordering problems using a primaldual interior point cutting plane method. Annals of Operations Research 62, 253-276 (1996)

29. Mitchell, J.E., Todd, M.J.: Solving combinatorial optimization problems using Karmarkar's algorithm. Mathematical Programming 56, 245-284 (1992)

30. Nunez, M.A., Freund, R.M.: Condition measures and properties of the central trajectory of a linear program. Mathematical Programming 83, 1-28 (1998)

31. Oskoorouchi, M.R., Ghaffari, H.R., Terlaky, T., Aleman, D.M.: An interior point constraint generation algorithm for semi-infinite optimization with health-care application. Operations Research 59(5), 1184-1197 (2011)

32. Righini, G., Salani, M.: New dynamic programming algorithms for the resource constrained elementary shortest path problem. Networks 51(3), 155-170 (2008)

33. Skajaa, A., Andersen, E., Ye, Y.: Warmstarting the homogeneous and self-dual interior point method for linear and conic quadratic problems. Mathematical Programming Computation pp. 1-25 (2012)

34. Solomon, M.M.: Algorithms for the vehicle routing and scheduling problems with time window constraints. Operations Research 35(2), 254-265 (1987)

35. Trigeiro, W.W., Thomas, L.J., McClain, J.O.: Capacitated lot sizing with setup times. Management Science 35(3), 353-366 (1989)

36. Wright, S.J.: Primal-Dual Interior-Point Methods. SIAM (1997)

37. Yildirim, E.A., Wright, S.J.: Warm-start strategies in interior-point methods for linear programming. SIAM Journal on Optimization 12(3), 782-810 (2002) 